\title{
Confined Migration Induces Heterochromatin Formation and Alters Chromatin Accessibility
} Chieh-Ren Hsia ${ }^{1,2}$, Jawuanna McAllister ${ }^{1,2}$, Ovais Hasan ${ }^{2}$, Julius Judd ${ }^{1}$, Seoyeon Lee ${ }^{3,4}$, Richa Agrawal ${ }^{1,2}$, Chao-Yuan Chang ${ }^{2,5}$, Paul Soloway ${ }^{3,4}$, Jan Lammerding ${ }^{2,5^{*}}$

1. Department of Molecular Biology and Genetics, Cornell University, Ithaca, NY, USA

2. Weill Institute for Cell and Molecular Biology, Cornell University, Ithaca, NY, USA

3. Department of Biomedical Sciences, Cornell University, Ithaca, NY, USA

4. Division of Nutritional Sciences, Cornell University, Ithaca, NY, USA

5. Nancy E. and Peter C. Meinig School of Biomedical Engineering, Cornell University, Ithaca, NY, USA

*Corresponding author: jan.lammerding@,cornell.edu

\begin{abstract}
During migration, cells often squeeze through small constrictions, requiring extensive deformation. We hypothesized that the nuclear deformation associated with such confined migration could alter chromatin organization and function. Studying cells migrating through collagen matrices and microfluidic devices that mimic interstitial spaces in vivo, we found that confined migration results in increased H3K9me3 and H3K27me3 heterochromatin marks that persist for several days. This "confined migration-induced heterochromatin" $(\mathrm{CMiH})$ was distinct from heterochromatin formation during migration initiation. $\mathrm{CMiH}$ predominantly decreased chromatin accessibility at intergenic regions near centromeres and telomeres, suggesting heterochromatin spreading from existing heterochromatin sites. Consistent with the overall decrease in chromatin accessibility, global transcription was decreased during confined migration. Intriguingly, we also identified increased accessibility at promoter regions of genes linked to chromatin silencing, tumor invasion, and DNA damage response. Inhibiting $\mathrm{CMiH}$ reduced migration speed, suggesting that $\mathrm{CMiH}$ promotes confined migration. Together, our findings indicate that confined migration induces chromatin changes that regulate cell migration and other cellular functions.
\end{abstract}




\section{Main text}

\section{Introduction}

Cell migration is a crucial biological process required for many physiological functions ${ }^{1,2}$. Cell migration also plays a pivotal role in metastasis, which is the major cause of cancer-related death ${ }^{3,4}$. In vivo, cells must often squeeze through narrow interstitial spaces that are only 1-20 $\mu \mathrm{m}$ in diameter, and thus substantially smaller than the size of the cell ${ }^{5,6}$. This recognition has led to an increased interest in studying cell migration in 'confined' environments, including the use of three-dimensional (3D) in vitro models, such as collagen matrices and microfluidic devices that resemble cell migration in vivo ${ }^{7}$. Previous studies have revealed that the nucleus, which is the largest and most rigid organelle ${ }^{8,9}$, undergoes severe deformation during confined migration $^{10-12}$. The nuclear deformation and physical stress associated with confined migration can result in nuclear envelope rupture and cause DNA damage ${ }^{10-13}$. Furthermore, recent evidence suggests that migration through confined spaces can lead to rearrangements in 3D genome organization in neutrophils and cancer cells $^{14,15}$, which could affect their transcriptional regulation and cellular functions. However, the effect of confined migration on chromatin modifications and its functional consequences have not been explored.

Chromatin is found in two distinct states: the relaxed and transcriptionally active euchromatin, and the condensed and transcriptionally silenced heterochromatin ${ }^{16,17}$. These states are controlled by posttranslational histone modifications ${ }^{18}$ and DNA methylation ${ }^{19}$. For example, trimethylation of lysine 9 on histone $\mathrm{H} 3$ (H3K9me3) is associated with constitutive heterochromatin, which mostly locates in centromeric and telomeric DNA regions ${ }^{16,17}$; trimethylation of lysine 27 on histone $\mathrm{H} 3$ (H3K27me3) is associated with facultative heterochromatin, which is developmentally-regulated ${ }^{16,17}$. In contrast, acetylation of lysine 9 on histone $\mathrm{H} 3(\mathrm{H} 3 \mathrm{~K} 9 \mathrm{ac})$ is associated with euchromatin and active promoters ${ }^{20}$. Increased H3K9me3 and H3K27me3 chromatin modifications are crucial to initiate 2D cell migration and for transwell migration ${ }^{21-}$ ${ }^{25}$, potentially by repression of specific genes ${ }^{26}$. At the same time, increased euchromatin facilitates cell migration through $3 \mathrm{D}$ collagen matrices ${ }^{27,28}$, likely by increasing nuclear deformability ${ }^{28,29}$. Intriguingly, recent studies have found that the physical microenvironment of cells can cause changes in chromatin modifications: mechanical compression of cells induces reversible chromatin condensation with increased H3K9me3 and H3K27me3 marks ${ }^{30}$, whereas cyclic stretching of cells leads to rapid and transient loss of heterochromatin ${ }^{31}$. Therefore, we aimed to explore whether nuclear deformation during confined migration could result in altered chromatin modifications, genomic accessibility, and transcriptional activity, and whether such changes could modulate the ability of cells to migrate through confined $3 \mathrm{D}$ environments.

We found that confined 3D migration in collagen matrices and microfluidic devices induced persistent global heterochromatin formation in cancer cells and fibroblasts, well beyond the changes observed during unconfined migration. The increased heterochromatin formation was dependent on histone modifying enzymes and modulated by nuclear envelope proteins and stretch-sensitive ion channels. Assay for Transposase-Accessible Chromatin using sequencing (ATAC-seq) analysis revealed that confined migration led to reduced chromatin accessibility at intergenic regions near centromeres and telomeres. Global reduction of transcriptional activity after confined migration further supports the predominantly repressing accessibility change. On the other hand, we identified increased chromatin accessibility at promoter regions of genes responsible for a wide range of pathways, such as chromatin silencing, tumor invasion, and DNA damage response. Preventing heterochromatin formation using histone methyltransferase inhibitors resulted in impaired migration, particularly through small constrictions, suggesting that confined migration-induced heterochromatin promotes confined migration. 
bioRxiv preprint doi: https://doi.org/10.1101/2021.09.22.461293; this version posted September 23, 2021. The copyright holder for this preprint

(which was not certified by peer review) is the author/funder. All rights reserved. No reuse allowed without permission.

\section{Results}

\section{Heterochromatin increases during and after cell migration through confined collagen matrices}

To investigate the effect of confined cell migration and the associated nuclear deformation on histone modifications, we studied HT1080 fibrosarcoma cells migrating through 3D collagen matrices of different collagen concentrations in the absence or presence of a broad-band matrix metalloproteinase (MMP) inhibitor. When MMPs are inhibited, cells must migrate through pre-existing pores in the collagen matrix, whose size correlates with the collagen concentration ${ }^{32}$. We chose collagen concentrations that result in average pore sizes ranging from $\approx 27 \mu \mathrm{m}^{2}(0.3 \mathrm{mg} / \mathrm{ml})$ to $\approx 4 \mu \mathrm{m}^{2}(1.7 \mathrm{mg} / \mathrm{ml})$ in cross section ${ }^{10,32}$. Cells were immunofluorescently stained for heterochromatin marks (H3K27me3 and H3K9me3) and a euchromatin mark (H3K9ac) (Fig. 1). To distinguish between true changes in chromatin modifications and effects of physical compression of the nuclear content due to deformation, we normalized the heterochromatin mark intensity to the euchromatin mark intensity in each cell. Increased heterochromatin formation should result in an increased ratio of heterochromatin marks to euchromatin marks, whereas physical compression of chromatin would increase both marks, and thus not alter their ratio. This ratio of heterochromatin marks to euchromatin marks represents the normalized heterochromatin level, which we refer to as "heterochromatin level" throughout the article.

Cells migrating in the collagen matrices had increased H3K27me3 heterochromatin levels with increasing collagen concentrations, corresponding to increased heterochromatin levels with decreasing pore size (Fig. 1a, b). Cells showed similar increases in the H3K9me3 constitutive heterochromatin levels with decreasing pore size (Fig. 1c, d), albeit to a slightly lower extent than for the facultative heterochromatin (H3K27me3). Surprisingly, treatment with an MMP inhibitor (MMPi) had little effect on heterochromatin levels when compared to vehicle-treated cells (Fig. 1b, d), suggesting that even when cells can degrade the collagen matrix, the matrix may still impose some confinement to the cells, although we cannot rule out that collagen concentrations could have confinement-independent effects on heterochromatin formation. Supporting the hypothesis that heterochromatin formation is linked to nuclear deformation and increased confinement, cells squeezing through tight pores in high concentration collagen matrices often showed locally enriched heterochromatin, mostly located in nuclear blebs (Supplementary Fig. 1), similar to the previously described heterochromatin enrichment in nuclear abnormalities (HENA) ${ }^{33}$. Human skin fibroblasts migrating in collagen matrices showed similar increase in heterochromatin with increasing confinement (Suppl. Fig. 2), indicating that the observed effect is not specific to cancer cells.

\section{Heterochromatin increases during and after cell migration through confined microfluidic channels}

To control the pore sizes that cells encounter during 3D migration independent of the collagen concentration, we performed migration experiments using custom-made polydimethylsiloxane (PDMS) microfluidic devices with precisely defined constrictions that mimic interstitial spaces (Fig. 2a, b). ${ }^{10,34}$ Cells migrated either through channels containing three rows of $\leq 2 \times 5 \mu \mathrm{m}^{2}$ constrictions that require substantial nuclear deformation to squeeze through ("confined channels"), or through $15 \times 5 \mu \mathrm{m}^{2}$ "control channels" that do not require substantial nuclear deformation for transit. Cells were categorized according to their locations in the device: 1) in the unconfined area "before" entering the channels, 2) in the middle of "squeezing" through the confined constrictions (or passing through the larger control constrictions), or 3) "after" migration out of the channels into the unconfined area (Fig. 2b, c, e). Migration of HT1080 cells resulted in increased H3K27me3 heterochromatin levels as the cells squeezed through the constrictions, and an even larger increase in cells that had exited the microfluidic channels (Fig. 2d). Staining for H3K9me3 showed similar results, although the facultative heterochromatin (H3K27me3) levels increased more substantially (Fig. 2e, f), consistent with the 3D collagen matrix migration results. Migration of skin fibroblasts and MDA-MB- 
231 breast cancer cells through the device yielded similar results (Suppl. Fig. 3a-f). Cells exhibited a progressive increase of heterochromatin levels as they migrated through the device (Fig. 2g; Suppl. Fig. 3g), whereas the intensity of the H3K9ac euchromatin mark remained constant or even decreased (Suppl. Fig. $3 \mathrm{~h}-\mathrm{m})$, further supporting accumulation of heterochromatin marks in response to confined migration. Since heterochromatin-associated histone modifications such as $\mathrm{H} 3 \mathrm{~K} 9 \mathrm{me} 3$ can lead to the recruitment of DNA methylation machinery ${ }^{19}$, we hypothesized that the increased heterochromatin histone marks would coincide with an increase in DNA methylation following migration through the devices. Indeed, migration through both control and confined channels increased 5-methylcytosine (5-meC) intensities (Suppl. Fig. 3n, o).

Surprisingly, even cells migrating through the $15 \times 5 \mu \mathrm{m}^{2}$ control channels displayed an increase in heterochromatin levels. Although the effect was less pronounced than in the $\leq 2 \times 5 \mu \mathrm{m}^{2}$ confined channels (Fig. 2c-f), it suggests that the vertical confinement of $5 \mu \mathrm{m}$ is sufficient to induce a nuclear response, consistent with recent observations that cell confinement to heights below 5 to $7 \mu \mathrm{m}$ is sufficient to trigger mechanosensing responses of the nucleus. ${ }^{35,36}$ To determine whether the observed increase in heterochromatin was due to physical confinement or merely represented chromatin changes associated with migration initiation ${ }^{22}$, we conducted experiments in which cells migrated either through the $15 \times 5 \mu \mathrm{m}^{2}$ control channels or through taller $\left(15 \times 10 \mu \mathrm{m}^{2}\right)$ channels that do not result in vertical confinement of the nucleus (Fig. 2h). Cells migrating through the 5- $\mu \mathrm{m}$ tall channels exhibited a significantly larger increase in heterochromatin than cells migrating through the $10-\mu \mathrm{m}$ tall channels (Fig. $2 \mathrm{~h}, \mathrm{i}$ ), demonstrating that the observed effect is primarily attributed to the confinement and not the migration process per se. Collectively, these data suggest that confined migration can result in increased and persistent heterochromatin formation, which we termed "confined migration-induced heterochromatin" (CMiH).

\section{Heterochromatin formation is induced after nuclear transit and is persistent after confined migration}

Since immunofluorescence staining is limited to end-point measurements, we performed time-lapse experiments with cells stably expressing GFP-HP1 $\alpha$, which binds to chromatin containing both H3K9me3 and H3K27me3 modifications ${ }^{37,38}$. We frequently observed local and persistent enrichments of GFP-HP1 $\alpha$ in nuclei during cell migration through confined channels compared to control channels (Fig. $2 \mathrm{j}, \mathrm{k}$ ). The GFP-HP1 $\alpha$ enrichments lasted for more than four hours after nuclear transit through the confined constrictions (Fig. 2j; Suppl. Fig. 4a, b; Suppl. Video 1), suggesting that the increase in GFP-HP1 $\alpha$ signal reflects persistent chromatin modifications and not transient pooling of mobile GFP-HP1 $\alpha$. To further validate that the confined migration-induced GFP-HP1 $\alpha$ enrichment reflects true heterochromatin formation, we performed fluorescence recovery after photobleaching (FRAP) analysis ${ }^{39}$ on the GFP-HP1 $\alpha$ enriched nuclear regions, with centromeric GFP-HP1 $\alpha$ foci serving as positive controls (Suppl. Fig. 4c-h). Nuclear blebs with enriched GFP-HP1 $\alpha$ signals had a decreased fraction of mobile GFP-HP1 $\alpha$ compared to nonbleb nuclear regions (Suppl. Fig. 4h), suggesting that these enrichments reflect GFP-HP1 $\alpha$ bound to heterochromatin formed in nuclear bleb regions.

To assess the persistence of $\mathrm{CMiH}$, we performed long-term time-lapse imaging of cells in the migration device in an incubator-mounted microscope combined with subsequent immunofluorescence analysis for H3K27me3 and H3K9ac histone marks (Suppl. Fig. 5a, b; Suppl. Video 2), enabling us to correlate heterochromatin levels with the time after nuclear transit through the constrictions for individual cells. Intriguingly, the heterochromatin levels in cells that had passed through the confined channels remained significantly elevated compared to cells in the unconfined "before" area during the 5-day observation period (Fig. 21). Cells maintained CMiH even after completing at least one round of mitosis, without any trend of reversion in their heterochromatin levels (Suppl. Fig. 5c, d), suggesting that the epigenetic modifications were inheritable through DNA replications. Cells that did not undergo mitosis after nuclear transit even showed a trend towards further increase in heterochromatin levels overtime (Suppl. Fig. 5e, f). Overall, these experiments indicate that $\mathrm{CMiH}$ persists for at least 5 days, even in proliferating cells. 


\section{Confined migration-induced heterochromatin formation is dependent on histone modifying enzymes}

To investigate the molecular mechanisms behind $\mathrm{CMiH}$ formation, we inhibited specific steps of the euchromatin-heterochromatin transition (Fig. 3a). Histone methyltransferases are required for the addition of methyl groups to H3K9 and H3K27. Treatment with 3-Deazaneplanocin A (DZNep), a broadband histone methyltransferase inhibitor (HMTi) ${ }^{29,40}$ (Suppl. Fig. 6a), significantly reduced CMiH (Fig. 3b), confirming that the observed changes in $\mathrm{H} 3 \mathrm{~K} 27 \mathrm{me} 3$ and $\mathrm{H} 3 \mathrm{~K} 9 \mathrm{me} 3$ heterochromatin levels were the result of increased histone methylation. We used a broadband histone deacetylase inhibitor (pan-HDACi), Trichostatin A (TSA), ${ }^{41}$ to block the removal of histone acetylation (Suppl. Fig. 6a), a pre-requisite for methylation of the same residues. Pan-HDACi treatment significantly reduced $\mathrm{CMiH}$ compared to vehicle controls (Fig. 3c), suggesting that the process is dependent on HDACs. One particular HDAC family member, HDAC3, had previously been implicated in catalyzing heterochromatin formation when cells are subjected to external compression or constraint spreading ${ }^{30,42}$. Thus, we hypothesized that HDAC3 may also play a crucial role in $\mathrm{CMiH}$ formation. Indeed, treatment with RGFP966, a potent HDAC3 inhibitor (HDAC3i) ${ }^{43}$ (Suppl. Fig. 6b), demonstrated nearly the same inhibitory effect on CMiH as pan-HDACi (Fig. 3d). Depletion of HDAC3 by siRNA (Suppl. Fig. 6c) similarly reduced $\mathrm{CMiH}$ after migration through confined channels, compared to non-target siRNA control (Suppl. Fig. 6d). Combined treatment of HMTi and pan-HDACi completely eliminated $\mathrm{CMiH}$, while also reducing the baseline heterochromatin levels before migration (Fig. 3e), speaking to the pivotal role of HMTs and HDACs in chromatin modifications. To test if the transition from heterochromatin back to euchromatin by histone demethylases (HDMs) also affects the levels of $\mathrm{CMiH}$, we used JIB-04, a Jumonji-domain histone demethylases inhibitor ${ }^{44}$ (HDMi) (Suppl. Fig. 6e). As expected, HDMi treatment increased $\mathrm{CMiH}$ in cells squeezing through constrictions and after migrating out of the channels, compared to vehicle controls (Fig. 3f).

\section{Confined migration-induced heterochromatin formation is controlled by HDAC3 phosphorylation}

To investigate the molecular mechanism responsible for $\mathrm{CMiH}$ in more detail, we examined the intracellular localization of HDAC3 during confined migration, as previous studies had reported that mechanical stimulation can trigger cytoplasmic to nuclear translocation of HDAC3 to induce heterochromatin formation $^{30,42}$. However, live cell microscopy of cells stably expressing GFP-HDAC3 did not reveal any changes in the nucleoplasmic-to-cytoplasmic (Nuc/Cyto) ratio of GFP-HDAC3 after nuclear transit through confined constrictions (Fig. 3g; Suppl. Video 3), suggesting that nuclear translocation of HDAC3 does not play a major role in $\mathrm{CMiH}$ formation. An alternative hypothesis is that confined migration results in increased activation of HDAC3, thereby contributing to increased heterochromatin formation. To test this hypothesis, we stained for phosphorylation of HDAC3 on Serine 424 (pSer424-HDAC3), which is critical for HDAC3 enzymatic activity ${ }^{45}$. Cells migration through confined channels, but not the larger control channels, led to increased levels of pSer424-HDAC3 (Fig. 3h). Since this particular HDAC3 phosphorylation is catalyzed by Casein Kinase $2(\mathrm{CK} 2)^{45}$, we tested whether depletion of CK2 $\alpha$, a key subunit of $\mathrm{CK}^{46}$ (Suppl. Fig. 6f), could inhibit $\mathrm{CMiH}$ formation. We observed reduced $\mathrm{CMiH}$ after cells depleted of CK2 $\alpha$ migrated through confined channels, when compared to non-target siRNA control (Fig. $3 i)$. However, the effect was mild and was not mirrored in cells squeezing through constrictions. This suggests that either residual $\mathrm{CK} 2$ levels are sufficient for $\mathrm{CMiH}$, or that additional, yet to be determined mechanisms regulating HDAC3 activity are involved in $\mathrm{CMiH}$ formation.

\section{Stretch-sensitive ion channels, but not nuclear envelope rupture, contribute to confined migration- induced heterochromatin formation}


To identify additional molecular players involved in $\mathrm{CMiH}$ formation, we investigated the role of the nuclear envelope (NE) proteins emerin and lamin A/C. HDAC3 localization to the nuclear periphery plays a crucial role in maintaining repression for specific gene loci during differentiation ${ }^{47}$, and HDAC3 binding to the NE protein emerin activates HDAC3's enzymatic activity and promotes its localization to the nuclear lamina ${ }^{48}$. However, depletion of emerin by siRNA (Suppl. Fig. 7a) did not reduce $\mathrm{CMiH}$ after migration, but instead resulted in increased heterochromatin levels as cells were squeezing through the constrictions (Suppl. Fig. 7b). Similarly, depletion of the NE proteins lamin A/C (Suppl. Fig. 7c), which play important roles in chromatin organization and help recruit emerin to the inner nuclear membrane ${ }^{47,49}$, did not reduce $\mathrm{CMiH}$ but increased the heterochromatin levels during severe nuclear deformation of cell squeezing (Suppl. Fig. $7 \mathrm{~d}$ ). As lamin $\mathrm{A} / \mathrm{C}$ and emerin have pleiotropic effects on chromatin organization and dynamics ${ }^{50-52}$, the exact mechanisms by which lamin $\mathrm{A} / \mathrm{C}$ and emerin affect heterochromatin levels during cell squeezing remain to be explored.

Recent studies found that stretch-sensitive ion channels can modulate heterochromatin changes in response to mechanical challenges ${ }^{29,31}$. To test if $\mathrm{CMiH}$ is dependent on stretch-sensitive ion channels, we treated cells with gadolinium chloride $\left(\mathrm{GdCl}_{3}\right)$, a broad inhibitor for stretch-sensitive ion channels ${ }^{53}$. $\mathrm{GdCl}_{3}$-treated cells had significantly reduced $\mathrm{CMiH}$ compared to vehicle treated controls (Fig. 4a), indicating that stretchsensitive ion channels contribute to $\mathrm{CMiH}$, for example, via influx of extracellular calcium and/or the release of intracellular calcium from the nuclear lumen and endoplasmic reticulum $(E R)^{35,36}$. A related mechanism may be the transient loss of NE integrity, termed "NE rupture", which is due to the physical stress on the nucleus during confined migration ${ }^{10-12}$. NE rupture could result in calcium release from the nuclear lumen and ER, as well as mislocalization of histone modifying enzymes, ions, or signaling molecules ${ }^{10-12}$, thereby leading to heterochromatin formation. To investigate the role of $\mathrm{NE}$ rupture in $\mathrm{CMiH}$, we performed timelapse migration experiments of cells expressing a fluorescent reporter (cGAS-mCherry) that accumulates at the site of NE rupture ${ }^{10}$, and then fixed the cells to stain for histone marks (Suppl. Fig. 7e). Notably, we did not detect any significant difference in $\mathrm{CMiH}$ between cells that incurred NE rupture during migration and those that did not (Fig. 4b; Suppl. Fig. 7f). Furthermore, cells that had experienced NE rupture did not show any significant correlation between the time that had passed since rupture and their heterochromatin levels (Fig. 4c; Suppl. Fig. 7g). Taken together, these data suggest that $\mathrm{CMiH}$ is independent of NE rupture.

\section{Confined migration alters chromatin accessibility}

Histone modifications often result in altered chromatin accessibility and gene expression. Increased chromatin accessibility near promoters allows binding of transcriptional machineries and regulatory elements, which can lead to increased gene expression ${ }^{54,55}$. In contrast, decreased chromatin accessibility is associated with silenced genomic regions and heterochromatin ${ }^{54,55}$. To study the effect of confined migration and $\mathrm{CMiH}$ on genome-wide chromatin accessibility, we performed Assay for Transposase-Accessible Chromatin using sequencing (ATAC-seq) ${ }^{56}$ analysis on cells that had migrated through collagen matrices with different collagen concentrations while inhibiting MMP activity (Fig. 5a), matching the experimental conditions from the experiments in which we had quantified global histone modifications by immunofluorescence staining (Fig. 1a-d).

Principal component analysis (PCA) of the ATAC-seq results showed clustering of samples according to the collagen concentrations (Fig. 5b), suggesting the progressive effect of increasing collagen concentrations (and decreasing pore size) on chromatin accessibility. Differential accessibility analysis of high versus low concentration collagen samples revealed 534 differentially accessible (DA) peaks that were up-regulated in high concentration collagen samples (referred to as "up" peaks), and 1,319 down-regulated DA peaks ("down" peaks) in high concentration collagen samples (Fig. 5c, panel i). The larger number of "down" peaks (purple) compared to "up" peaks (red) indicates an overall reduction in chromatin accessibility, which is consistent with the globally increased $\mathrm{H} 3 \mathrm{~K} 27 \mathrm{me} 3$ and $\mathrm{H} 3 \mathrm{~K} 9 \mathrm{me} 3$ heterochromatin marks observed in cells 
migrating in high concentration collagen matrices (Fig. 1a-d). Other comparisons, i.e., high vs. medium and medium vs. low collagen concentrations, showed similar but less pronounced patterns, with a larger number of "down" than "up" peaks (Fig. 5c, panels ii-iii). A meta-analysis of all "down" peaks revealed that the ATAC-seq signal of these peaks remained low at all collagen concentrations, without large visual differences, despite their statistical significance (Fig. 5d, left panel), suggesting that these peaks correspond to genomic regions that are already silenced at baseline and thus only poorly accessible. In contrast, the ATAC-seq signal of "up" peaks progressively increased with increasing collagen concentration (Fig. 5d, right panel), suggesting that "up" peaks may be regulating genes activated by confined migration. As expected, when we quantified the genomic location annotation of these DA peaks, we found that the majority of "down" peaks were located at intergenic regions, whereas most of the "up" peaks were located at promoter-transcription start sites (TSS) of known genes (Fig. 5e).

\section{Confined migration decreases intergenic chromatin accessibility near centromeres and telomeres}

To further access the potential effects of chromatin accessibility changes on nearby genes, we plotted each peak's distance to the TSS of its associated genes (i.e., computationally mapped nearby genes and regulatory domains) using Genomic Regions Enrichment of Annotations Tool (GREAT) ${ }^{57}$. Most of the "down" peaks were located far (50-500 kb) from the TSS of the associated genes (Fig. 6a), consistent with their annotation as being located in "intergenic regions" (Fig. 5e). These data suggest that most of the computationally associated genes are unlikely to be regulated by "down" peaks, although in some cases, ATAC-seq signals of the "down" peaks mapped onto the corresponding genes displayed small but progressive decrease in accessibility from low to high collagen concentrations (Fig. 6b; Suppl. Table 1).

Our findings indicate that most "down" peaks are in genomic regions with low chromatin accessibility, i.e., regions that are likely already silenced by heterochromatin at baseline. We thus hypothesized that heterochromatin spreading from centromeric or telomeric regions plays a role in $\mathrm{CMiH}$ formation, since heterochromatin spreading from pre-existing heterochromatin is a common mechanism of heterochromatin formation $^{58,59}$. When we mapped the distance of each DA peak to the centromere or telomeres of the same chromosome, we found that many "down" peaks, but only few "up" peaks, were located within $3 \mathrm{Mb}$ of centromeres or telomeres (Fig. 6c), well within the $<9 \mathrm{Mb}$ definition of peri-centromeric regions or the $<7$ $\mathrm{Mb}$ definition of sub-telomeric regions ${ }^{60}$. When mapping peaks to whole chromosomes as karyoplots, "down" peaks were visually clustered within or near the centromeres and telomeres, whereas "up" peaks did not follow any obvious pattern (Fig. 6d; Suppl. Fig. 8a, b). Moreover, "down" peaks shared among all three different comparisons (high vs. low, high vs. medium, and medium vs. low) of collagen concentration samples (Suppl. Fig. 8c) had even more peaks within $3 \mathrm{Mb}$ of centromeres or telomeres than "down" peaks in high vs. low comparison only (Suppl. Fig. 8d), suggesting that $\mathrm{CMiH}$ formation consistently clustered around these constitutive heterochromatin sites.

Since centromeres, telomeres, and nearby regions contain an abundance of highly repetitive DNA sequences and transposable elements (TEs) ${ }^{61}$, which are not detectable by conventional sequencing analysis methods, we used custom-developed algorithms ${ }^{62,63}$ to map TEs to "up" or "down" peaks. As expected, "down" peaks were significantly enriched with TEs, including the cancer-overexpressed alpha satellite, ALR_Alpha ${ }^{64}$, and one of the most active TEs in the abundant Alu TE family, AluYj4 ${ }^{65}$, (Suppl. Fig. 8e, panel i). In contrast, "up" peaks were depleted for TEs (Suppl. Fig. 8e, panel ii). The locations of the enriched TEs matched centromeres or telomeres (Suppl. Fig. 8f, g), further strengthening the observation of "down" peaks clustering around these sites. Taken together, these data suggest that $\mathrm{CMiH}$ is associated with reduced chromatin accessibility near centromeres and telomeres, likely resulted from spreading of constitutive heterochromatin sites, rather than the silencing of new and gene-rich genomic regions. 


\section{Confined migration decreases global transcription and nascent mRNA levels}

Since the global increase in heterochromatin marks and the ATAC-seq analysis suggest a predominantly repressive effect of confined migration on gene expression, we investigated the impact of confined migration on RNA polymerase II phosphorylation (pSer2-RNAPII), a marker of active transcription elongation ${ }^{66}$. Consistent with the chromatin modifications, cells that had migrated through either the control or confined channels had lower pSer2-RNAPII levels than cells in the unconfined "before" area, but the effect was more pronounced in the confined channels (Fig. 6e), indicating that confined migration represses transcription. To directly visualize the relative level of transcription, we pulsed cells with 5-ethynyl uridine (5-EU) to label nascent mRNA transcripts ${ }^{67}$. The results revealed a significant decrease of nuclear 5-EU signal in cells squeezing through the constrictions and after migration through the control and confined channels (Fig. 6f), consistent with the reduced RNAPII phosphorylation and a decrease in transcription. The fact that even migration through the $15 \times 5 \mu \mathrm{m}^{2}$ control channel reduced transcription was consistent with our earlier finding that the $5 \mu \mathrm{m}$ channel height is sufficient for $\mathrm{CMiH}$ formation (Fig. $2 \mathrm{~h}$, i). The overall repressed transcription in cells migrating through $5-\mu \mathrm{m}$ tall channels supports the ATAC-seq results of highly repressive chromatin accessibility changes induced by confined migration, reflecting the silencing effect of $\mathrm{CMiH}$.

\section{Confined migration increases chromatin accessibility at genes associated with diverse cellular functions}

Given that a high fraction of "up" peaks locations were annotated as promoter-TSS regions (Fig. 5e), we examined their relationship to nearby genes. The peak-to-TSS distribution showed that most of the "up" peaks were located within $5 \mathrm{~kb}$ of the TSS of associated genes (Fig. 7a). Mapping the ATAC-seq signals of representative "up" peaks onto the corresponding genes revealed a progressive increase in accessibility near the genes' promoter-TSS from low to high concentration collagen (Fig. 7b; Suppl. Table 2). However, although statistically highly significant, the $\log _{2}$-fold changes $(\log 2 \mathrm{FC})$ of "up" peaks were typically small in magnitude, with the largest $\log _{2}$-fold changes only $\approx 0.4$ (Suppl. Table 1 ), suggesting that any associated transcriptional changes may be relatively small and difficult to detect. Gene Ontology (GO) Biological Process enrichment analysis of genes associated with "up" peaks (Table 1) showed an enrichment in chromatin/gene silencing and other chromatin-related functions (Suppl. Table 2), DNA damage checkpoint (Suppl. Table 3), and cell cycle checkpoint (Suppl. Table 4). Moreover, GO Cellular Component enrichment analysis revealed enrichment of DNA packaging complex, nucleosome, and chromosome (telomeric region) associated genes (Table 2). Interestingly, many of the annotated genes encode histones proteins, including genes from all four major histone families of $\mathrm{H} 2 \mathrm{~A}, \mathrm{H} 2 \mathrm{~B}, \mathrm{H} 3$ and $\mathrm{H} 4$, as well as several genes critical for heterochromatin formation and maintenance, such as histone acetyltransferase 1 (HAT1), HDAC3, and CBX5 (which encodes HP1 $\alpha$ ) (Suppl. Table 2).

Canonical pathway analysis of the nearest genes of "up" peaks using Ingenuity Pathway Analysis (IPA) ${ }^{68}$ identified a diverse list of significant pathways, including pathways related to cell migration, tumor invasion, histone methylation, and heterochromatin formation (Table 3). Moreover, several of the pathways, such as TGF- $\beta$ signaling, Pyridoxal 5'-phosphate Salvage Pathway and ERK5 Signaling, had previously been linked to migration-induced $\mathrm{H} 3 \mathrm{~K} 27 \mathrm{me} 3$ heterochromatin in scratch wound assays ${ }^{26}$. Predicted regulatory networks of "up" peak-associated genes generated using IPA identified cell migration or tumor invasion, heterochromatin formation, and cell growth among the activated downstream functions, whereas DNA damage was among the inhibited downstream functions (Fig. 7c; Suppl. Fig. 9). Moreover, genes associated with "up" peaks that were shared among different comparisons (high vs. low, high vs. medium, and medium vs. low) of collagen concentration samples (Suppl. Fig. 10a) exhibited the same predicted downstream functions (Suppl. Fig. 10b), suggesting that such gene functions are important for confined migration. Overall, the presence of numerous chromatin-related functions in the GO term and pathway analysis indicates that changes in chromatin accessibility caused by confined migration may drive further chromatin 
remodeling steps involved in $\mathrm{CMiH}$. Other cellular functions identified in the analysis are consistent with previous findings that cell migration activates tumor invasion and cell proliferation pathways ${ }^{26}$, and that migration through confined spaces can induce DNA damage pathways ${ }^{10,11,13,69}$ and alter cell cycle $\operatorname{progression}^{70,71}$.

To identify transcription factors (TFs) that may regulate "up" peaks in response to confined migration, we performed motif enrichment analysis on "up" peak sequences and matched the enriched DNA motifs to known TF binding motifs (Suppl. Fig. 10c). The identified TF included SP1 and SMAD3 (underlined in Suppl. Fig. 10c), which form a complex under TGF- $\beta$ signaling to regulate tumor progression and invasion $^{72,73}$. Intriguingly, SP1 was also a predicted upstream regulator (underlined in Fig. 7c), whereas SMAD3 gene was identified as part of the chromatin GO terms ( $\dagger$ in Suppl. Table 2) and one of the genes associated with the shared "up" peaks (highlighted in Suppl. Fig. 10b). These findings suggests that both SP1 and SMAD3 could serve potential roles as upstream TFs that regulate accessibility and downstream functions in genes associated with "up" peaks.

\section{Confined migration regulates co-accessibility of enhancer-promoter interactions}

One central mechanism by which chromatin accessibility changes can regulate gene expression is through enhancer-promoter interactions (EPIs) that exhibit co-accessibility, i.e., having the same direction of accessibility changes ${ }^{74,75}$. To identify potential EPIs in "up" and "down" peaks, we assigned peaks that were within $1 \mathrm{~kb}$ upstream of the nearest gene's TSS to be "promoter-associated", and all other peaks as "nonpromoter-associated". As expected, there were many more promoter-associated peaks in "up" peaks than in "down" peaks (Suppl. Fig. 11a). We then mapped all peaks to the DNase I hypersensitive regions in the ENCODE database ${ }^{76}$, as DNase I hypersensitivity validates chromatin regulatory elements, including promoters and enhancers 55 . "Up" peaks overlapped much more with the DNase I data, suggesting that "up" peaks contained more putative promoters and enhancers than "down" peaks (Suppl. Fig. 11b). Based on the validated peaks, we found that EPI distance (promoter to non-promoter, peak-to-peak distance) was significantly longer for "up" peaks, compared to "down" peaks (Suppl. Fig. 11c). This finding suggests that long-range (i.e., more than a few hundreds of $\mathrm{kb}$ ) enhancer-mediated regulation plays a role in "up" peaksassociated genes, which is a sign of epigenetic dysregulation and compromised chromatin domains in cancer $^{77}$. We identified 41 and 7 EPIs in "up" peaks and "down" peaks, respectively, based on the conventional $500 \mathrm{~kb}$ cutoff $\mathrm{f}^{78}$ (Suppl. Fig. 11d). Representative EPIs in "up" peaks mapped to the enhancer and promoter database annotations (Suppl. Fig. 11f), supporting the validity of our EPI identification. Overall, these identified EPIs suggest that confined migration-induced chromatin accessibility changes may influence gene expression via enhancer-mediated regulation.

\section{Heterochromatin formation promotes confined migration}

The significant extent of $\mathrm{CMiH}$ formation, the associated chromatin accessibility changes, and the predicted downstream functions prompted us to investigate effects of $\mathrm{CMiH}$ on migration. Previous studies showed that reducing heterochromatin levels by treatment with pan-HDACi (TSA) or HMTi (5'-deoxy-5'methylthioadenosine) impairs migration through $3 \mathrm{D}$ confined environments ${ }^{79,80}$. Since reduced nuclear stiffness via lamin depletion facilitates nuclear transit through confined spaces ${ }^{81,82}$, it is puzzling that the reduced nuclear stiffness due to lowered heterochromatin level would have an opposite effect on confined migration $^{80}$. Therefore, we decided to test if $\mathrm{CMiH}$ offered an advantage for cell migration through confined channels. HMTi (DZNep) treatment resulted in increased nuclear transit time (i.e., decreased migration speed) through constrictions in both control and confined channels compared to vehicle controls, but the effect was more pronounced in confined channels (Fig. 7d; Suppl. Videos 4-7). The observed effect of HMTi on confined migration speed was consistent with previous studies ${ }^{79,80}$, demonstrating the importance of 
$\mathrm{CMiH}$ for efficient nuclear transit through tight spaces. On the other hand, increasing heterochromatin during confined migration via HDMi (JIB-04) treatment did not affect confined migration speed (Fig. 7e). However, under conditions that slow down migration, such as mild hypothermia $\left(33^{\circ} \mathrm{C}\right)$, which inhibits cancer cell proliferation and migration ${ }^{83,84}$, HDMi reduced nuclear transit time through constrictions in confined channels compared to vehicle controls (Fig. 7f; Suppl. Videos 8, 9). These data suggest that additional heterochromatin formation does not speed up confined migration in general, but that it can promote confined migration under adverse conditions. Overall, our data show that $\mathrm{CMiH}$ is crucial for efficient confined migration, and additional heterochromatin can promote confined migration under environmental conditions adverse for cell migration.

\section{Discussion}

In this study, we demonstrated that $\mathrm{CMiH}$ is a prominent feature across cancer and non-cancerous cell lines. Confined migration led to increases in $\mathrm{H} 3 \mathrm{~K} 27 \mathrm{me} 3$ and H3K9me3 levels, both globally and in nuclear blebs, but H3K27me3 increased more substantially, resonating with previous findings of its importance in cancer cell migration ${ }^{23,26}$. Vertical confinement to $5 \mu \mathrm{m}$ height was sufficient to induce $\mathrm{CMiH}$, consistent with previous studies on the nuclear mechanosensing threshold being around 5 to $7 \mu \mathrm{m}^{35,36}$, and $\mathrm{CMiH}$ increased further with increasing confinement and with migration through successive constrictions. While we cannot completely rule out that the observed chromatin changes are permissive for confined migration and thus cells with these features are selected for during confined migration, time-lapse microscopy revealed formation of persistent GFP-HP1 $\alpha$ enrichments in cells during and after nuclear transit through tight constrictions, indicating that this is an active process during confined migration. Taken together, these findings indicate that $\mathrm{CMiH}$ is distinct from heterochromatin associated with general migration initiation, which occurs prior to the onset of migration in $2 \mathrm{D}$ and $3 \mathrm{D}$ environments ${ }^{22,26}$.

Intriguingly, $\mathrm{CMiH}$ persisted for at least 5 days and was inheritable after mitosis. One possible consequence of these increased heterochromatin levels is replication stress and subsequent DNA damage ${ }^{85-87}$. In support of this hypothesis, DNA damage checkpoint and cell cycle checkpoint were among the enriched GO terms associated with increased chromatin accessibility during confined migration. On the other hand, nuclear deformation during confined migration is sufficient to cause replication stress and DNA damage ${ }^{13}$, which could result in heterochromatin formation ${ }^{88}$. Thus, confined migration-induced DNA damage could be both upstream and downstream of $\mathrm{CMiH}$.

We identified that $\mathrm{CMiH}$ is dependent on a combination of histone modifying enzymes, such as HDAC3, and stretch-sensitive ion channels. However, it is unclear if redundant mechanosensing pathways exist for $\mathrm{CMiH}$ regulation, such as through the deformation of nuclear-ER membrane and the subsequent calcium ion influx ${ }^{35,36}$, or through force transmission via cytoskeletons and the linker of nucleoskeleton and cytoskeleton (LINC) complexes ${ }^{89,90}$.

Since the majority of heterochromatin readouts in this study were based on immunofluorescence staining at a fixed time point, one limitation is the lack of real-time data tracking single cells throughout the migration process with quantifiable reporters such as fluorescence resonance energy transfer (FRET) biosensor ${ }^{91}$ or mintbodies $^{92}$ that reports chromatin modification changes. However, such single-cell fluorescence-based methods are very sensitive to the intracellular distribution of the reporter, changes in $\mathrm{pH}$, imaging settings, and phototoxicity/photobleaching during repeated imaging, making them challenging to work with for longterm time-lapse microscopy studies of migrating cells.

Our ATAC-seq studies suggest that the increase in global heterochromatin marks predominantly corresponds to spreading of existing heterochromatin marks in intergenic or transcriptionally repressed regions. The functional consequences of these modifications could include both changes in the mechanical 
properties of the nucleus such as nuclear compaction and stiffening ${ }^{29,80}$, but also transcriptional activity due to decreased enhancer accessibility ${ }^{74,75}$. On the other hand, we also identified numerous genes with increased accessibility of their promoter-TSS regions. These genes, which frequently had GO terms and pathways associated with chromatin modifications and tumor cell invasion, could promote both the formation of $\mathrm{CMiH}$ and downstream effects of confined migration on other cellular functions. Furthermore, coaccessibility analysis of the ATAC-seq data demonstrated that confined migration may regulate multiple EPIs that leads to gene expression changes, and long-range EPIs may play a role in the regulation of genes with increased accessibility (i.e., genes associated with "up" peaks). However, since there were far less "up" peaks than "down" peaks, the distances between "up" peaks were overall longer (Suppl. Fig. 11e), potentially resulting in a bias toward overall longer EPI distances in "up" peaks, compared to "down" peaks.

Importantly, we found that $\mathrm{CMiH}$ promotes confined migration, as preventing $\mathrm{CMiH}$ using $\mathrm{HMTi}$ significantly increased transit time through tight spaces. Possible mechanisms for the pro-migration effect of $\mathrm{CMiH}$ include changes in gene expression, nuclear biophysical properties, or a combination of both. For example, $\mathrm{CMiH}$ may repress unwanted transcriptional activities, and in turn promote confined migrationrelated gene expression, similar to the role of $\mathrm{H} 3 \mathrm{~K} 27 \mathrm{me} 3$ in $2 \mathrm{D}$ migration initiation ${ }^{26}$. On the other hand, $\mathrm{CMiH}$ may alter nuclear stiffness and nuclear size ${ }^{29,80}$, which were shown to affect nuclear transit speed through confined spaces ${ }^{80,82}$. Intriguingly, nuclear size was increased under HMTi treatment (Suppl. Fig. 12a) and decreased under HDMi treatment (Suppl. Fig. 12b). The exact mechanism by which $\mathrm{CMiH}$ promotes confined migration, however, will require further studies beyond the scope of this work.

In conclusion, we revealed the phenomenon of heterochromatin formation in the context of confined 3D migration, while providing insights on its impact on cell migration and the molecular mechanisms behind it. We have already demonstrated the presence of $\mathrm{CMiH}$ in tumor cells and fibroblasts but hypothesize that $\mathrm{CMiH}$ also occurs in other cell types, making it relevant for immune responses, wound healing, and during development. Furthermore, the recognition of $\mathrm{CMiH}$ in confined migration may provide motivation for future research to target this mechanism to reduce or prevent cancer metastasis. Moreover, our findings could potentially connect the role of chromatin to the role of the nucleus as a cellular mechanosensor ${ }^{35,36}$, and help pave the way for future studies on how chromatin modifications could affect nuclear and cellular responses to the cell's physical microenvironment. 


\section{Methods}

\section{Cell lines and cell culture}

The fibrosarcoma cell line HT1080 (ACC315) was purchased from DSMZ in Braunschweig, Germany. The breast adenocarcinoma cell line MDA-MB-231 (ATCC HTB-26) was purchased from American Type Culture Collection (ATCC). The SV40-immortalized human fibroblasts were purchased from the Coriell Institute for Medical Research. All cell lines were cultured in Dulbecco's Modified Eagle Medium (DMEM, Gibco) supplemented with 10\% (v/v) fetal bovine serum (FBS, Seradigm) and 1\% (v/v) penicillin and streptomycin (Pen-Strep, Gibco) (i.e., complete DMEM), in the incubator under humidified conditions at $37^{\circ} \mathrm{C}$ and $5 \% \mathrm{CO}_{2}$.

\section{Generation of fluorescently labeled cell lines}

HT1080 cells and MDA-MB-231 cells were stably or transiently modified with either of the following constructs: lentiviral construct GFP-HP1 $\alpha$ (pCDH-CMV-EGFP-HP1 $\alpha$-EF1-puro) for imaging of heterochromatin formation; retroviral construct NLS (nuclear localization sequence)-GFP and H2BtdTomato (pQCXIP-NLS-copGFP-P2A-H2B-tdTomato-IRES-puro) for measuring of nuclear transit time ${ }^{93}$; GFP-HDAC3 (pCMV-EGFP-HDAC3, a gift from Nikhil Jain) for imaging of HDAC3 translocation; lentiviral construct cGAS-mCherry (pCDH-CMV-cGAS ${ }^{\mathrm{E} 225 \mathrm{~A} / \mathrm{D} 227 \mathrm{~A}}$-mCherry2-EF1-blastiS) for imaging of NE rupture ${ }^{10}$.

\section{Viral modification}

Pseudo-viral particles were produced as described previously ${ }^{10}$. In brief, 293-TN cells (System Biosciences) were co-transfected with lentiviral packaging plasmid and envelope plasmid (psPAX2 and pMD2.G, gifts from Didier Trono) using PureFection (SBI), following the manufacturer's protocol. Lentivirus-containing supernatants were collected at 48 hours and 72 hours post-transfection and filtered. Cells were seeded into 6-well plates to reach 50-60\% confluency on the day of infection and were transduced 2-3 consecutive days with the viral supernatant in the presence of $8 \mu \mathrm{g} / \mathrm{mL}$ polybrene (Sigma). The viral supernatant was replaced with fresh culture medium, and cells were cultured for 24 hours before selection with $1 \mu \mathrm{g} / \mathrm{mL}$ of puromycin (InvivoGen) or $6 \mathrm{ug} / \mathrm{mL}$ of blasticidine $\mathrm{S}$ (InvivoGen) for 7 days.

\section{siRNA-mediated depletion}

siRNAs (small interfering RNAs) used were as followed: human LMNA (ON-TARGET plus SMARTpool, Dharmachon Horizon, L-004978-00), human EMD (Ambion Silencer Select, ID: s4646), human HDAC3 (ON-TARGET plus SMARTpool, Dharmachon Horizon, L-003496-00), human CK2 $\alpha$ (Santa Cruz, sc29918), and non-target (NT) negative control (ON-TARGETplus non-targeting pool, Dharmachon, D001810-10). Cells were seeded into 12-well plates at a density of 40,000 cells per well the day prior to treatment, and transfected with siRNA using Lipofectamine RNAiMAX (Invitrogen) following the manufacturer's protocol at a final concentration of $20 \mathrm{nM}$. Cells were transfected again with fresh siRNA at 24 hours. Cells were trypsinized for migration experiments at 48 hours after transfection. For HDAC3 knockdown, $40 \mathrm{nM}$ of siRNA was used, and cells were trypsinized for migration experiments at 72 hours after transfection, including 24 hours of recovery in culture media without transfection reagents. Cells treated with the same condition and duration were harvested for validation of successful protein depletion by Western blot analysis and immunofluorescence (IF) staining. 
Cells were treated with inhibitors at the time of seeding into the migration device and throughout the experiment. Fresh culture media containing inhibitors were changed every 24 hours. Cells were treated with either $5 \mu \mathrm{M}$ of 3-Deazaneplanocin A (DZNep, Cayman Chemical) for broad-band inhibition of histone methyltransferases (with the exception of $10 \mu \mathrm{M}$ for HT1080 cells staining experiments); $250 \mathrm{nM}$ of Trichostatin A (TSA, Sigma) for broad-band inhibition of HDACs (with the exception of $125 \mathrm{nM}$ for MDAMB-231 cells staining experiments); $10 \mu \mathrm{M}$ of RGFP966 (Selleckchem) for inhibition of HDAC3; $1 \mu \mathrm{M}$ of JIB-04 (Selleckchem) for inhibition of Jumonji histone demethylases; $10 \mu \mathrm{M}$ of $\mathrm{GdCl}_{3}$ (Santa Cruz) for inhibition of mechanosensitive ions channels. Cells treated with the same condition and duration were harvested for validation of successful enzymatic activity inhibition by Western blot analysis and IF staining. Inhibitor stocks were dissolved in dimethyl sulfoxide (DMSO) (Sigma) (with the exception of $\mathrm{GdCl}_{3}$ in water) before diluting into cell culture medium.

\section{Single cell collagen matrix migration assays}

To create glass-bottom wells for collagen matrices, blocks of polydimethylsiloxane (PDMS) were punched with a $10 \mathrm{~mm}$ biopsy punch and covalently bonded onto glass coverslips (VWR) after plasma treatment of 5 minutes. The preparation and making of collagen gel matrices was done as described previously ${ }^{10,94}$. In short, individual wells on glass coverslips were treated with $1 \%$ polyethylenimine (PEI, Sigma), followed by $0.1 \%$ glutaraldehyde (Sigma) treatment for consistent bonding of collagen matrices, and washed with PBS. To generate single cell-containing collagen matrices at specified collagen concentrations $(0.3,1.0$, and $1.7 \mathrm{mg} / \mathrm{mL}$ ), acidic solution of rat tail type-I collagen (Corning) was supplemented with complete DMEM and $\mathrm{NaOH}$ to reach a neutral $\mathrm{pH}$ of 7.4, and then mixed with cell suspension to reach a final density of 100,000 cells per $\mathrm{mL}$. Collagen matrices were allowed to polymerize in the incubator for 30 min before adding complete DMEM with either DMSO or a board MMP inhibitor, GM6001 (Millipore, $20 \mu \mathrm{M}$ ) to submerge the matrices. Cells were allowed to migrate in the matrices for 48 hours before fixation and IF staining.

\section{Fabrication and use of microfluidic migration devices}

The microfluidic migration devices were designed and fabricated as described previously ${ }^{10,34}$. In brief, PDMS replicas of the migration device molds were made from Sylgard 184 following the manufacturer's protocol $(1: 10)$ and baking at $65^{\circ} \mathrm{C}$ for 2 hours. Once the PDMS is demolded and cut into individual blocks of devices, biopsy punches were used to create reservoirs and cell seeding pores. Glass coverslips (VWR) were cleaned with $0.2 \mathrm{M} \mathrm{HCl}$ overnight, rinsed with water and isopropanol, and dried with compressed air. Covalent bonding protocol: For live cell imaging experiments, the migration devices were assembled after plasma treatment of both the PDMS blocks and glass coverslips for 5 minutes, by gently pressing the PDMS blocks on the activated coverslips to form covalent bonds. The assembled devices were heated on a hot plate at $95^{\circ} \mathrm{C}$ for 5 minutes to improve adhesion.

Non-covalent bonding protocol: For fixed cell IF staining experiments, the migration devices were assembled after plasma treatment of only the glass coverslips for 5 minutes, by gently pressing the PDMS blocks on the activated coverslips to form non-covalent bonds. The assembled devices were heated on a hot plate at $65^{\circ} \mathrm{C}$ for 1 hour to improve adhesion, and then plasma treated for another 5 minutes after prolonged (10 minutes) vacuuming.

After bonding, devices were filled with $70 \%$ ethanol (the step was skipped in non-covalent bonding protocol) to sterilize, then rinsed and coated with $50 \mu \mathrm{g} / \mathrm{mL}$ rat tail type-I collagen (Corning) in $0.02 \mathrm{~N}$ acetic acid overnight at $4^{\circ} \mathrm{C}$. After coating, the devices were rinsed with complete DMEM before cell seeding. For overnight live cell imaging experiments and standard IF staining experiments, 30,000 cells suspended in 5 $\mu 1$ of media were seeded into individual loading chambers of the migration devices. A 0 -to- $200 \mathrm{ng} / \mathrm{mL}$ platelet-derived growth factor (PDGF, Cell Signaling) gradient in DMEM was established for migration of human fibroblasts. For low confluency IF staining experiments (5-days-long time-lapse imaging, staining 
with inhibitor treatments and siRNA depletions), 6,000 cells were seeded, and a 2-to-10\% (v/v) FBS gradient in DMEM was established for increased cell migration capacity. Subsequently, devices were put in the incubator, for either a minimum of 6 hours to allow cell adhesion before live cell imaging under a LSM700 confocal microscopy (Zeiss), or around 48 hours to allow cell migration before fixation and IF staining. For live cell imaging, cells were cultured and imaged in FluoroBrite DMEM (Gibco) supplemented with 10\% FBS, 1\% Pen-Strep, GlutaMax (Gibco) and $25 \mathrm{mM}$ HEPES (Gibco). Where indicated, media inside the devices was supplemented with DMSO (vehicle) or inhibitors throughout the experiments. For experiments with GFP-HDAC3, SPY555-DNA (Cytoskeleton, 1:3,000) was used to label nuclear regions.

\section{Immunofluorescence staining}

For staining of cells in microfluidic migration devices, cells were fixed with 4\% paraformaldehyde (PFA, Sigma) in PBS for 30 minutes at room temperature. After PBS washes, the devices were carefully removed by inserting a razor blade from the PDMS edges into the interface between the PDMS and the glass coverslips, and then gradually lifting up the PDMS blocks. For staining of cells seeded directly on glass coverslips, cells were fixed with 4\% PFA in PBS for 10 minutes at room temperature. The fixation step was followed by permeabilization with $0.3 \%$ Triton-X100 (Sigma) in PBS for 15 minutes, blocking with 3\% bovine serum albumin (BSA) (Sigma) in PBS supplemented with $0.2 \%$ Triton-X100 and 0.05\% Tween-20 (Sigma) for 1 hour at room temperature, and incubation overnight at $4{ }^{\circ} \mathrm{C}$ with primary antibodies (rabbit anti-H3K9me3, Abcam, ab8898, 1:1,000; rabbit anti-H3K27me3, Millipore, 07-449, 1:250; mouse antiH3K9ac, GeneTex, GT464, 1:500; mouse anti-HDAC3, Cell Signaling, 3949, 1:200 ; rabbit anti-pSer424HDAC3, Cell Signaling, 3815, 1:200; mouse anti-lamin A/C, Santa Cruz, sc-376248, 1:300; mouse antilamin A, Santa Cruz, sc-518013, 1:100; mouse anti-emerin, Leica Biosystems, NCL-EMERIN, 1:200, rabbit anti-pSer2 RNA polymerase II, Abcam, ab5095, 1:500) in the same blocking buffer. The samples were then washed with PBS and stained with Alexa Fluor secondary antibodies (Invitrogen) and DAPI (Sigma, 1:1,000). For staining of 5-methylcytosine, cells were treated with $4 \mathrm{~N} \mathrm{HCl}$ for an hour at $37^{\circ} \mathrm{C}$ for denaturing of DNA, and washed with PBS before blocking and primary antibody incubation (mouse-anti 5methycytosine, Eurogentec, BI-MECY-0100, 1:250). After PBS washes, the samples were mounted with Hydromount Nonfluorescing Mounting Media (Electron Microscopy Sciences) onto glass slides (VWR) for confocal microscopy imaging.

\section{5-ethynyl uridine labeling}

To label nascent mRNA, cells were pulsed and labeled as previously described ${ }^{67}$, and following manufacturer's protocols. Cells migrating in microfluidic devices were pulsed with $1 \mathrm{mM} 5$-ethynyl uridine (EU, Jena Bioscience) in complete DMEM for 4 hours, and then fixed with 4\% PFA in PBS for 30 minutes. After two PBS washes, the devices were carefully removed as described in the IF staining section. The cells were then permeabilized with $0.3 \%$ Triton-X100 (Sigma) in PBS for 15 minutes, and incubated for 30 minutes with freshly prepared EU labeling buffer containing $100 \mathrm{mM}$ Tris base (Sigma), $100 \mu \mathrm{M}$ Alexa Fluor 488 conjugated-azide (Invitrogen), $4 \mathrm{mM} \mathrm{CuSO}_{4}$ (Sigma), and $100 \mathrm{mM}$ ascorbic acid (Sigma) in water. After the incubation, cells were washed with PBS, and another round of 30 minutes incubation with EU labeling buffer and PBS washes was repeated. Cells were then incubated with primary antibodies and proceeded with the IF staining protocol.

\section{Protein immunoblot analysis}

Cells were lysed in RIPA buffer or high-salt RIPA buffer supplemented with cOmplete EDTA-Free protease inhibitor (Roche) and PhosSTOP phosphatase inhibitor (Roche) on ice for 3 minutes. Lysates were then vortexed for 5 minutes, and spun down at $4{ }^{\circ} \mathrm{C}$. For lysates blotting for proteins that form network scaffolds (lamin A/C and emerin), lysates were sonicated and briefly boiled before spinning down to remove potential DNA contamination. Supernatant were then transferred to new tubes. Protein concentration was quantified 
by Bio-Rad Protein Assay and a Model 550 Microplate Reader. $25 \mu \mathrm{g}$ of protein lysate was heat-denatured at $95^{\circ} \mathrm{C}$ for 5 minutes in Laemmli sample buffer (Bio-Rad), and then separated using a 4 to $12 \%$ Bis-Tris polyacrylamide gel (Invitrogen) following a standard SDS-PAGE protocol. Protein was transferred using a semi-dry system (Bio-Rad) to a polyvinylidene fluoride membrane (Millipore, IPVH00010) for 1.5 hour at a current of $16 \mathrm{~mA}$. Membranes were blocked with 3\% BSA in Tris-buffered saline containing $0.1 \%$ Tween20 (TBST) for 1 hour at room temperature, and then incubated overnight at $4{ }^{\circ} \mathrm{C}$ with primary antibodies (rabbit anti-H3K9me3, Abcam, ab8898, 1:3,000; rabbit anti-H3K27me3, Millipore, 07-449, 1:3,000; mouse anti-H3K9ac, GeneTex, GT464, 1:3,000; mouse anti-lamin A/C, sc-376248, Santa Cruz, 1:1,000; mouse anti-emerin, Leica Biosystems, NCL-EMERIN, 1:500; mouse anti-HDAC3, Santa Cruz, sc-136290, 1:500; mouse anti-CK2 $\alpha$, Santa Cruz, sc-365762, 1:250) in the same blocking buffer. After TBST rinses, membranes were incubated with loading control primary antibody (mouse anti-histone H3, Abcam, ab195277, 1:5,000; rabbit anti-histone H3, Cell Signaling, 4499, 1:1,000; mouse anti-GAPDH, Proteintech, 60004-1-Ig, 1:20,000; rabbit anti-pan-actin, Cell Signaling, 8456, 1:1,000) for 1 hour at room temperature. Protein bands were detected using IRDye 680LT and IRDye 800CW secondary antibodies (LI-COR), imaged with an Odyssey CLx imaging system (LI-COR), and analyzed with Image Studio Lite (LI-COR).

\section{Extended imaging using an incubator microscope}

Long-term imaging was performed using an IncuCyte Live-Cell Analysis System (Sartorius) inside the cell culture incubator. Cells expressing NLS-GFP and migrating inside the microfluidic migration devices were imaged using the IncuCyte filter module for 5 days, every 10 minutes, with a $20 \times$ objective. The acquired migration movies were processed and exported using the IncuCyte ZOOM software. At the end of imaging, the cells were immediately fixed for IF staining of heterochromatin and euchromatin marks.

\section{Confocal microscopy}

Fixed cells on coverslips and live cells migrating in microfluidic devices were imaged with an inverted Zeiss LSM700 confocal microscope. Z-stack images were collected using $20 \times$ air $(\mathrm{NA}=0.8)$ or $40 \times$ waterimmersion $(\mathrm{NA}=1.2)$ objectives. Airy units for all images were set at 1.0. For overnight live cell imaging, the image acquisition was automated through ZEN (Zeiss) software at 10 minutes intervals, at $37^{\circ} \mathrm{C}$ in a heated stage chamber for 12-16 hours. Where indicated, temperature of the heated chamber was lowered to $33^{\circ} \mathrm{C}$.

\section{Fluorescence recovery after photobleaching (FRAP)}

Images were acquired with an inverted Zeiss LSM700 confocal microscope using a $\times 63$ oil-immersion $(\mathrm{NA}=1.4)$ objective, at $37^{\circ} \mathrm{C}$ in a heated stage chamber. Three $1.8 \mu \mathrm{m}$ by $1.8 \mu \mathrm{m}$ regions were selected in each cell: one as the "unbleached" control region, one as the low GFP-HP1 $\alpha$ intensity "euchromatin" control region, and one as the high GFP-HP1 $\alpha$ intensity "inquiry" region (with GFP-HP1 $\alpha$ foci or enrichment). The three regions were first imaged for 2 seconds at $1 \%$ power of $488 \mathrm{~nm}$ laser and 0.2 second intervals, to calculate the pre-bleach values. The euchromatin control and the inquiry regions were then bleached with a laser pulse of $12 \%$ power for 20 iterations. Following bleaching, the three regions were imaged for at least 24 seconds at $1 \%$ power and 0.2 second intervals. The FRAP image acquisition was automated through ZEN (Zeiss) software. Fluorescence recovery was measured as percentage of recovery (with pre-bleach value as $100 \%$ ), normalized to unbleached control. Immobile fractions were measured as fluorescence intensity that had not recovered after 24 seconds, after the recovery curve had reached plateau (by around 22 seconds).

Assay for Transposase-Accessible Chromatin using sequencing (ATAC-seq) 
ATAC-seq was done using cells collected after 48 hours of migration in collagen matrices at specified collagen concentrations (low: $0.3 \mathrm{mg} / \mathrm{mL}$; medium: $1.0 \mathrm{mg} / \mathrm{mL}$; high: $1.7 \mathrm{mg} / \mathrm{mL}$ ), following Omni-ATAC protocol ${ }^{56}$ with custom modifications. Scripts used to perform the analyses including exact parameters can be accessed at https://github.com/jaj256/ConfinedATAC. Please refer to Supplementary Information for detailed methods of ATAC-seq sample preparation, library preparation and analysis pipeline.

\section{Image analysis}

Image sequences were analyzed using ZEN (Zeiss), ImageJ or MATLAB (Mathworks) using only linear intensity adjustments uniformly applied to the entire image region. Region-of-interest intensities were extracted using ZEN or ImageJ. All confocal image stacks were three-dimensionally reconstructed as maximum intensity projections, and then processed with standard background subtraction (rolling ball). To quantify normalized heterochromatin values, nuclear areas were selected by thresholding using the medianfiltered DAPI channel. Mean grey intensity of the heterochromatin mark (H3K9me3 or H3K27me3) and the euchromatin mark (H3K9ac) were quantified, and the heterochromatin intensity was divided by the euchromatin intensity to obtain normalized heterochromatin values. Apoptotic fragments and overlapped nuclei were manually excluded from the dataset. Experiments with high variability of cell seeding between control and experimental groups were excluded. For all other quantification of staining intensities, nuclear areas were selected by thresholding using the median-filtered DAPI channel (or comparable nuclear signal when DAPI was not available), and mean grey intensities of channels of interests were quantified. All staining thresholding and quantification were performed through custom ImageJ Macros (available at https://github.com/chiehrenhsia/ConfinedMigration) to ensure consistency and reproducibility. For generation of normalized heterochromatin images, the heterochromatin mark channel (H3K9me3 or $\mathrm{H} 3 \mathrm{~K} 27 \mathrm{me} 3$ ) was divided by the euchromatin mark (H3K9ac) channel using the "image calculator" function in ImageJ. In the euchromatin $(\mathrm{H} 3 \mathrm{~K} 9 \mathrm{ac})$ channel, pixels with 0 value were converted to 1 to ensure division without errors. The normalized image was then median-filtered and a nuclear mask of median-filtered DAPI channel was applied to exclude artifacts of non-nuclear background noise. To quantify cells with persistent GFP-HP1 $\alpha$ enrichments, time-lapse cell migration movies were blinded to the observer to record the time duration of GFP-HP1 $\alpha$ enrichment formation after the start of nuclear transit in Rainbow RGB pseudo-color channel. An enrichment was defined by at least $30 \%$ increase in nuclear bleb GFP intensity compared to the main nucleus. The durations of all GFP-HP1 $\alpha$ enrichments were plotted, and enrichments with durations longer than the mean $+95 \%$ confidence interval were considered significantly longer than the mean, therefore defined as persistent enrichments. To quantify GFP-HDAC3 nucleoplasmic-to-cytoplasmic (Nuc/Cyto) ratio, nuclear region was selected using SPY555-DNA signal, and whole-cell region was traced manually in ImageJ. Cytoplasmic GFP signal was calculated by whole-cell GFP signal subtracting nuclear GFP signal. Nuc/Cyto ratio was then calculated by dividing nuclear signal with cytoplasmic signal. Nuc/Cyto ratio of GFP-HDAC3 from 2-6 time points immediately before and after nuclear transit were quantified and averaged. To quantify nuclear transit time through microfluidic migration channels, timelapse cell migration movies were analyzed using a custom MATLAB program, as described previously ${ }^{93}$. Any single outlier with a transit time 180 minutes greater than the rest of the dataset was excluded in each experiment. Graphs were generated in Prism 7.0a or 8.2.0 (GraphPad) or Excel (Microsoft), and figures were assembled in Illustrator (Adobe).

\section{Statistical analysis}

Unless otherwise noted, all experimental results are pooled from at least three independent experiments. For data with normal distribution, two-tailed Student's $t$ test with Welch's correction (comparing one variable in two conditions), or one-way analysis of variance (ANOVA) with Tukey's post hoc test for multiple comparisons (comparing one variable in more than two conditions) was used. For data where two factors were involved, two-way ANOVA with Tukey's post hoc test for multiple comparisons was used. For data with non-normal distribution, Wilcoxon Rank Sum test (comparing one variable in two conditions), or 
Kruskal-Wallis H test with Dunn's post hoc test for multiple comparisons (when two factors were involved) was used. For paired data (Nuc/Cyto ratio of GFP-HDAC3 before and after nuclear transit), paired $t$ test was used. All statistical tests were performed using Prism version 7.0a for Mac, or version 8.2.0 for Windows (GraphPad). Statistical details are provided in the figure legends. Unless otherwise noted, error bars represent the standard error of the mean (SEM). 


\section{Acknowledgments}

The authors thank Cornell University's Transcriptional Regulation \& Expression Facility (TREx), especially Jen Grenier, for ATAC-seq library preparation their help with the analysis; Cornell University's Biotechnology Resource Center (BRC) Genomics Facility for ATAC-seq library quality control and sequencing; Cornell University's Statistical Consulting Unit (CSCU) for suggestions and reviewing of statistical analysis. The authors thank Philipp Isermann, Tyler Kirby, Pragya Shah, and other members of the Lammerding group for their helpful discussions and support. This work was supported by awards from the National Institutes of Health (R01 HL082792, R01 GM137605, U54 CA210184 to J.L.), the Department of Defense Breast Cancer Research Program (Breakthrough Award BC150580 to J.L.), the National Science Foundation (CAREER Award CBET-1254846 to J.L.), the VolkswagenStiftung (to J.L.) and the Taiwanese Ministry of Education (GSSA-2017 to C.-R.H.). J.J. was supported by National Human Genome Research Institute fellowship (F31HG010820). The content is solely the responsibility of the authors and does not necessarily represent the official views of the National Institutes of Health. This work was performed in part at the Cornell NanoScale Science \& Technology Facility (CNF), a member of the National Nanotechnology Coordinated Infrastructure NNCI), which is supported by the National Science Foundation (Grant NNCI2025233).

\section{Author Contributions}

C.-R.H. and J.L. conceptualized and designed all experiments; C.-R.H., J.M., and O.H. performed experiments; J.J. and S.L. optimized the ATAC-seq analysis pipeline and performed the ATAC-seq analysis; R.A. designed and manufactured the $10-\mu \mathrm{m}$ tall microfluidic migration devices; C.-Y.C. optimized initial histone modifying enzyme inhibitor conditions; C.-R.H. and J.L. wrote the paper; all authors contributed to the editing of the manuscript; P.S. and J.L. acquired funding.

\section{Competing Interests}

The authors declare no competing interests.

\section{Data Availability}

ATAC-seq data that supports the findings of this study have been deposited in Gene Expression Omnibus (GEO) repository, with the accession number GSE181247. The other datasets generated during and/or analysed during the current study are available from the corresponding author (Jan Lammerding; jan.lammerding(cornell.edu) on reasonable request.

The ImageJ Macro scripts used to analyze confocal immunofluorescence staining images and to generate normalized heterochromatin images can be accessed at https://github.com/chiehrenhsia/ConfinedMigration. The R scripts used to perform ATAC-seq analyses including the exact parameters can be accessed at https://github.com/jaj256/ConfinedATAC. 


\section{Figure and Tables}

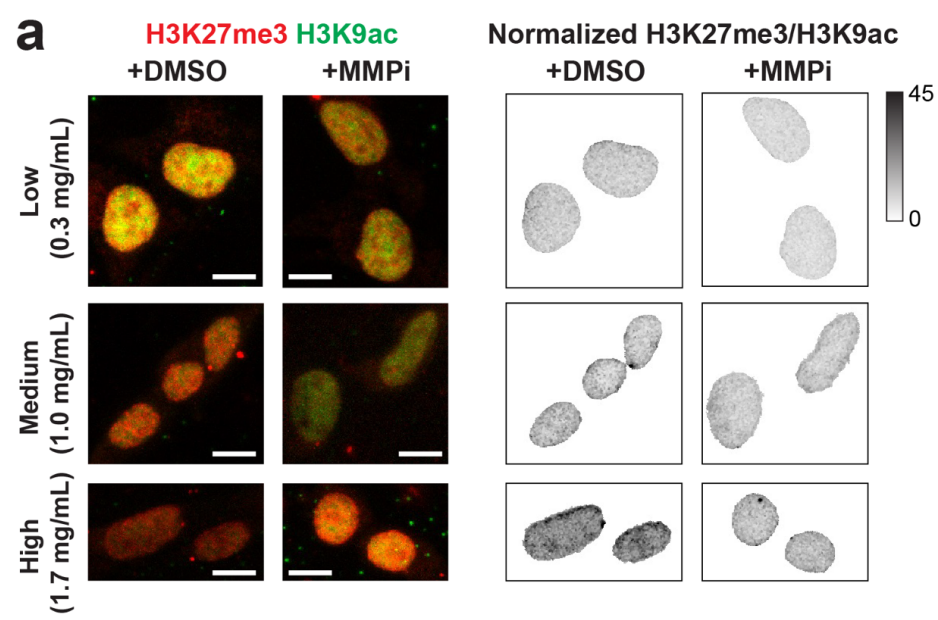

C

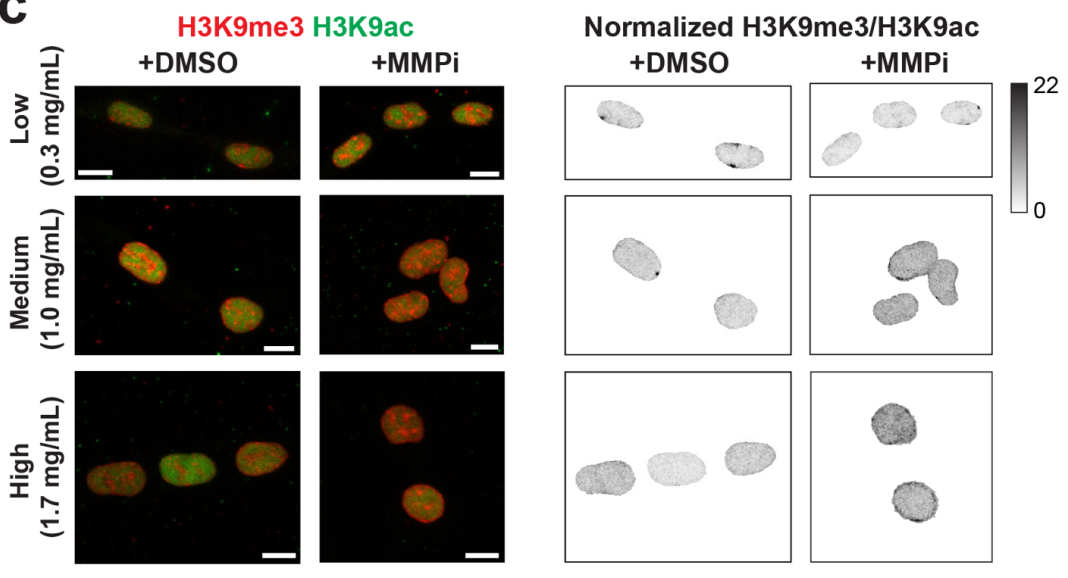

b

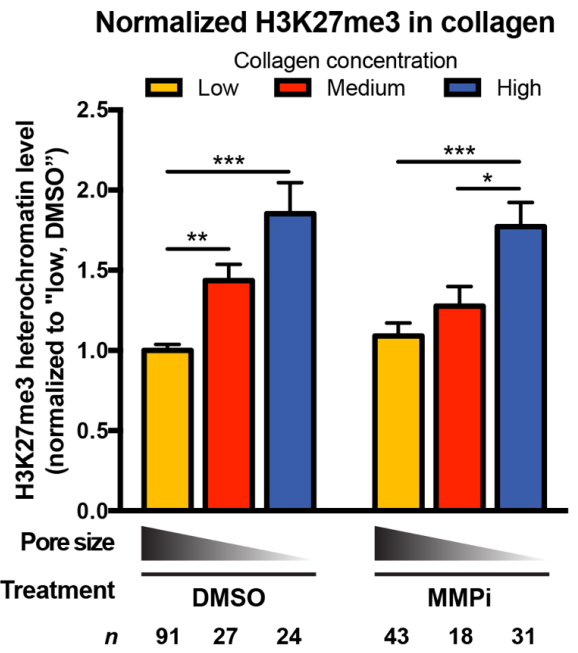

d

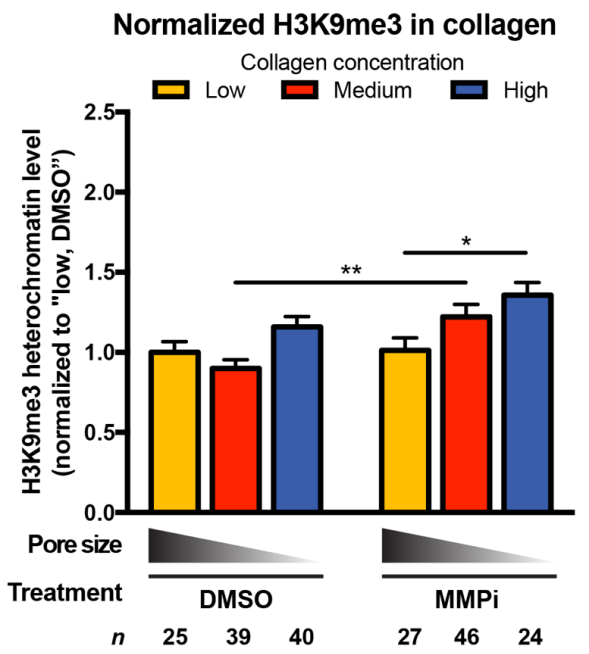

Figure 1: Confined migration induces heterochromatin formation in collagen matrices. (a) Representative staining of H3K27me3 (red) and H3K9ac (green) in HT1080 cells migrating in low (0.3 $\mathrm{mg} / \mathrm{mL})$, medium $(1.0 \mathrm{mg} / \mathrm{mL})$ or high $(1.7 \mathrm{mg} / \mathrm{mL})$ concentration collagen matrices, under DMSO (vehicle control) or MMPi treatment. Normalized heterochromatin level (H3K27me3 divided by H3K9ac) is shown in inverted grayscale. Scale bars:10 $\mu \mathrm{m}$. Grayscale bar (0-45, on the right): the range of normalized level. (b) Quantification of normalized H3K27me3 heterochromatin level in undeformed HT1080 cells migrating in collagen matrices, under DMSO or MMPi treatment. All values are normalized to DMSO-treated cells in low concentration collagen. ${ }^{*} p<0.05, * * p<0.01, * * * p<0.001$, two-way ANOVA with Tukey's multiple comparison test. (c) Representative staining of H3K9me3 (red) and H3K9ac (green) in HT1080 cells migrating in different concentrations of collagen matrices, under DMSO (vehicle control) or MMPi treatment. Normalized heterochromatin level (H3K9me3 divided by $\mathrm{H} 3 \mathrm{~K} 9 \mathrm{ac}$ ) is shown in inverted grayscale. Scale bars: $10 \mu \mathrm{m}$. Grayscale bar $(0-22$, on the right): the range of normalized level. (d) Quantification of normalized H3K9me3 heterochromatin level in undeformed HT1080 cells migrating in collagen matrices, under DMSO or MMPi treatment. All values are normalized to DMSO-treated cells in low concentration collagen. ${ }^{*} p<0.05,{ }^{*} p<0.01$, two-way ANOVA with Tukey's multiple comparison test. Data are presented as mean \pm SEM. 
a
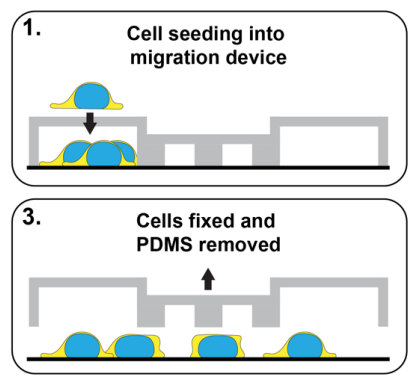

C
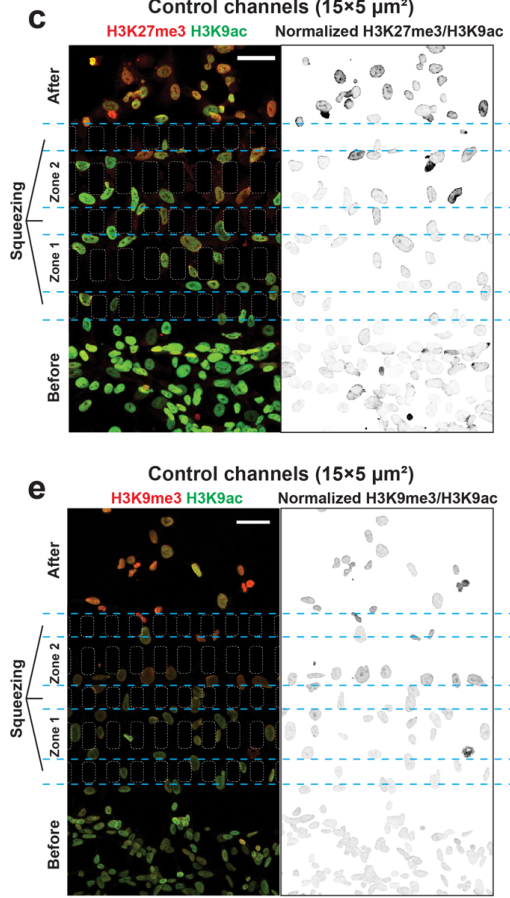

g

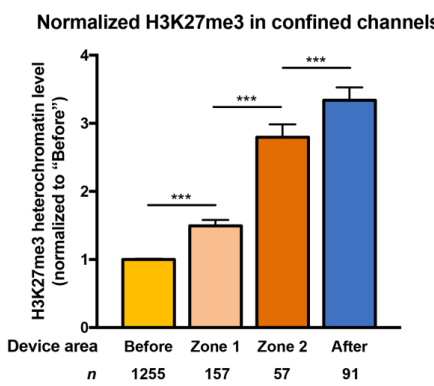

j

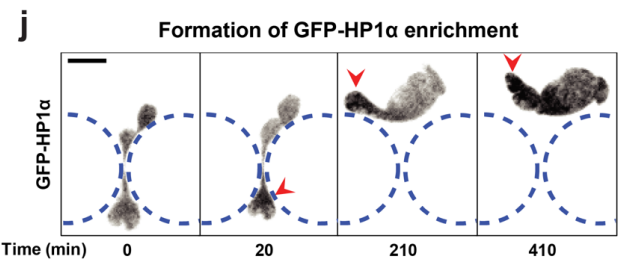

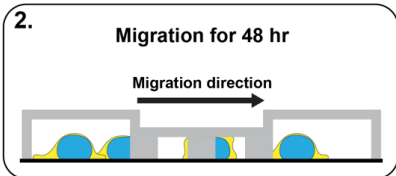

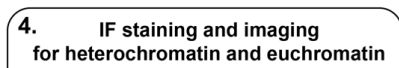
for heterochromatin and euchromatin

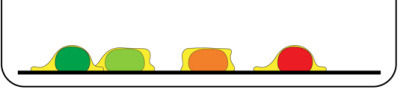

Confined channels $\left(\leq 2 \times 5 \mu \mathrm{m}^{2}\right)$
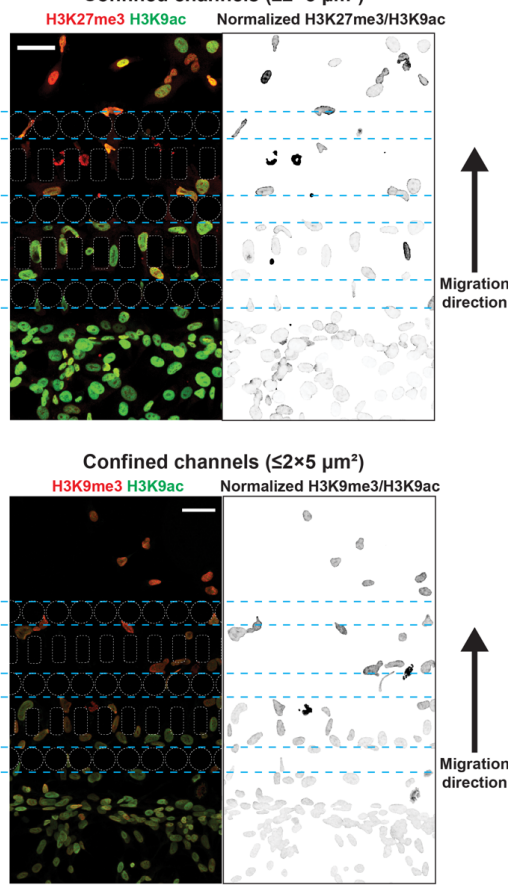

h

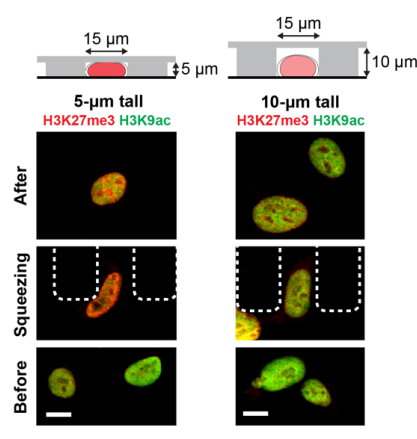

K Cells with GFP-HP1a enrichments

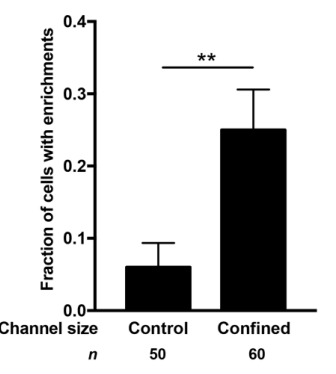

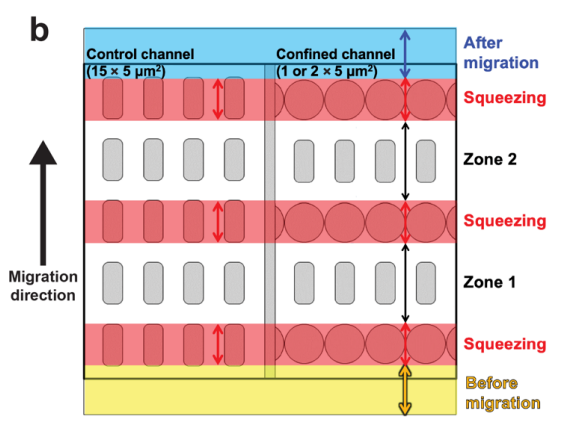

d

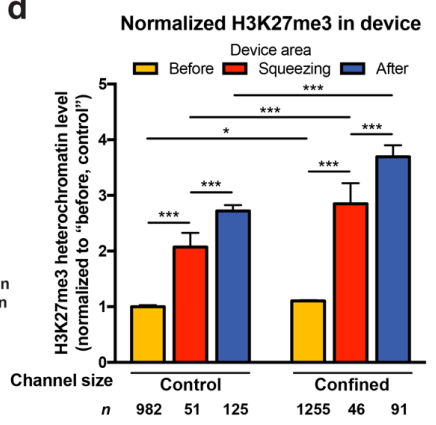

f

Normalized $\mathrm{H} 3 \mathrm{~K} 9 \mathrm{me} 3$ in device

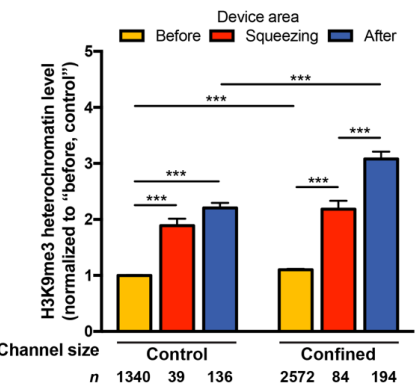

i

Normalized H3K27me3 in control channels

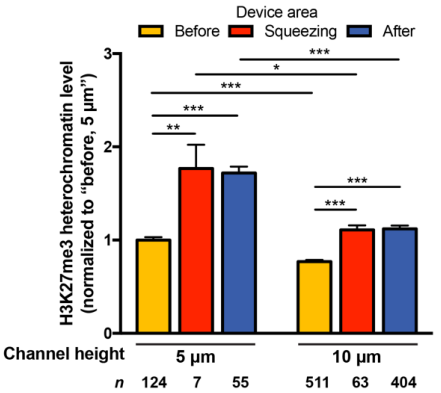

I

Figure 2: Confined migration induces heterochromatin formation in microfluidic migration devices. (a) The workflow of staining experiments using PDMS microfluidic migration devices. Unlabeled cells 
were seeded into the device (step 1). Cells were allowed migration for 48 hours (step 2) before fixation and removal of the PDMS (step 3), and the cells were stained for chromatin marks (step 4). (b) A simplified schematic illustrating the design and the different areas in the migration device. Yellow indicates the unconfined area "before" cells entering the device. Red indicates the area while cells are "squeezing" or passing through the constrictions. Blue indicates the unconfined area "after" cells migrating out of the channels. "Zone 1" indicates the area after cells made one nuclear transit through the first row of constrictions. "Zone 2" indicates the area after cells made two nuclear transits through the first two rows of constrictions. (c) Representative staining of H3K27me3 (red) and H3K9ac (green) in HT1080 cells migrating in a migration device, in control or confined channels. Normalized heterochromatin level is shown in inverted grayscale. Scale bars: $40 \mu \mathrm{m}$. (d) Quantification of normalized H3K27me3 heterochromatin level in HT1080 cells migrating in migration devices. All values are normalized to control channels "before" cells. ${ }^{*} p<0.05, * * * p<0.001$, two-way ANOVA with Tukey's multiple comparison test. (e) Representative staining of H3K9me3 (red) and H3K9ac (green) in HT1080 cells migrating in a migration device, in control or confined channels. Normalized heterochromatin level is shown in inverted grayscale. Scale bars: $40 \mu \mathrm{m}$. (f) Quantification of normalized H3K9me3 heterochromatin level in HT1080 cells migrating in migration devices. All values are normalized to control channels "before" cells. ${ }^{* * *} p<0.001$, two-way ANOVA with Tukey's multiple comparison test. (g) Comparison between normalized H3K27me3 heterochromatin level of HT1080 cells in each area of the device. All values are normalized to "before" cells. $* * * p<0.001$, oneway ANOVA with Tukey's multiple comparison test. (h) Representative staining of H3K27me3 (red) and H3K9ac (green) in HT1080 cells migrating in devices with $5 \mu \mathrm{m}$ or $10 \mu \mathrm{m}$-tall control channel. Crosssection view of channel design is shown above. Scale bars: $10 \mu \mathrm{m}$. (i) Quantification of normalized H3K27me3 heterochromatin level in HT1080 cells migrating in devices with $5-\mu \mathrm{m}$ or $10-\mu \mathrm{m}$ tall control channels. All values are normalized to $5 \mu \mathrm{m}$-tall control channels "before" cells. $* p<0.05, * * p<0.01,{ }^{* * *} p$ $<0.001$, two-way ANOVA with Tukey's multiple comparison test. (j) Representative inverted grayscale image sequence of local enrichment formation of GFP-HP1 $\alpha$ (arrowhead) in HT1080 cells. Scale bar: 10 $\mu \mathrm{m}$. (k) Quantification of HT1080 cells with local enrichment of GFP-HP1 $\alpha$ in control and confined channels. ${ }^{* *} p<0.01$, student's $t$ test with Welch's correction for unequal variances. (l) The correlation of normalized H3K27me3 heterochromatin level with time after the last nuclear transit through confined constrictions in HT1080 cells, binned by an interval of 24 hours. All values are normalized to "before" cells. $* * * p<0.001$, one-way ANOVA with Tukey's multiple comparison test. Data are presented as mean $\pm \mathrm{SEM}$. 
a

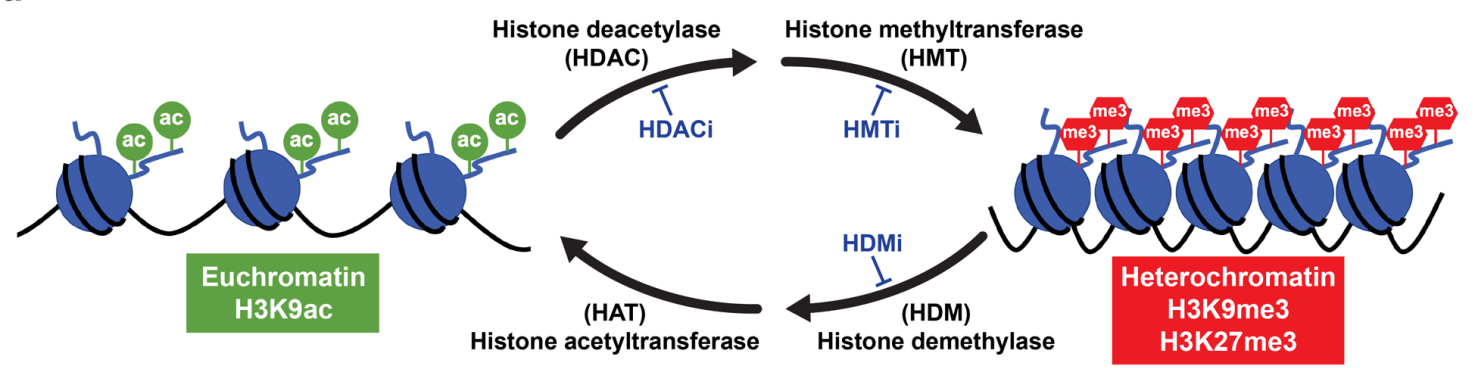

b

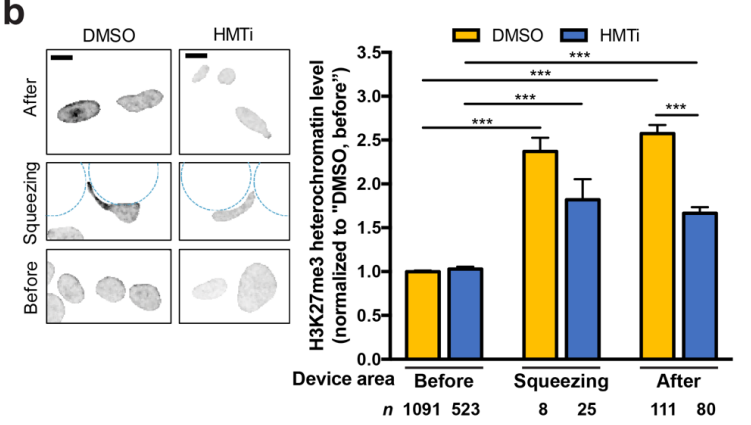

d

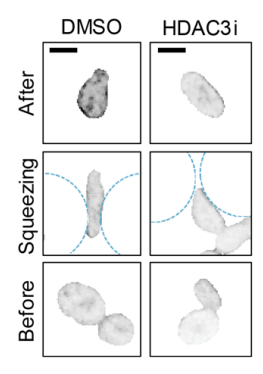

f
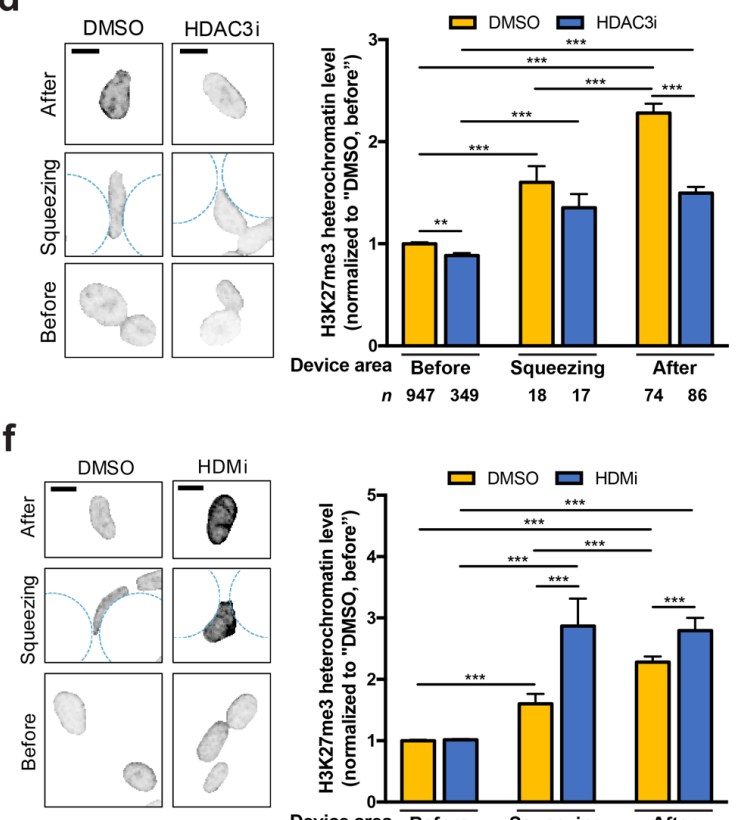

n

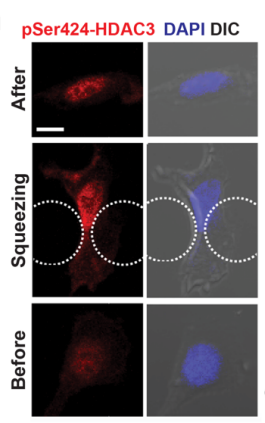

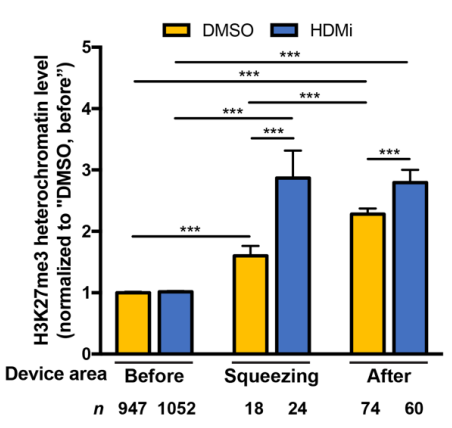

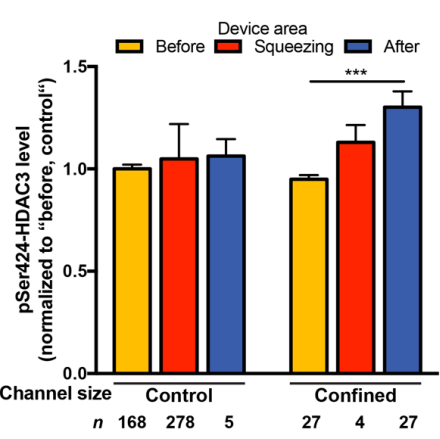

C
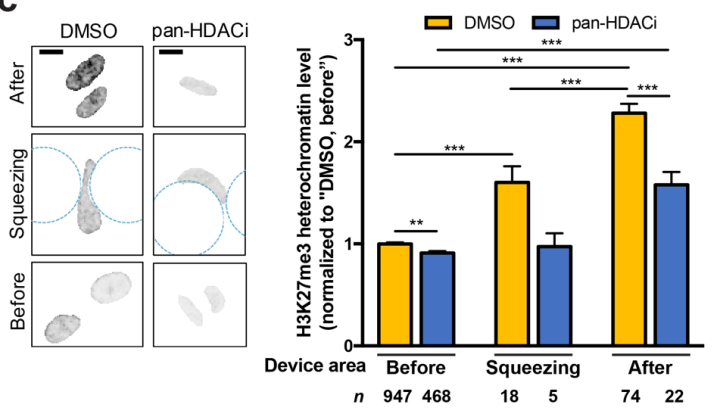

e

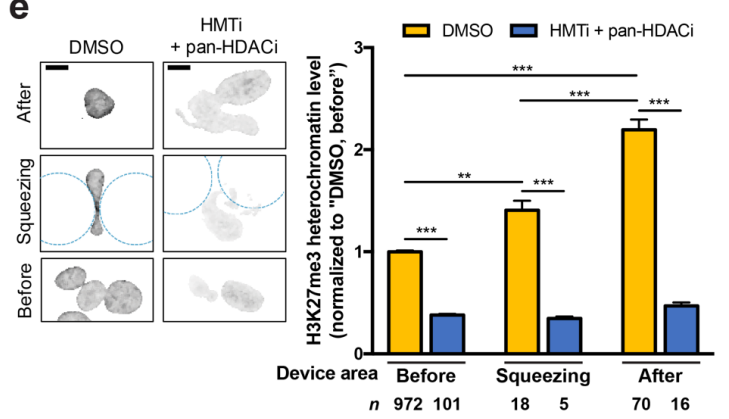

g
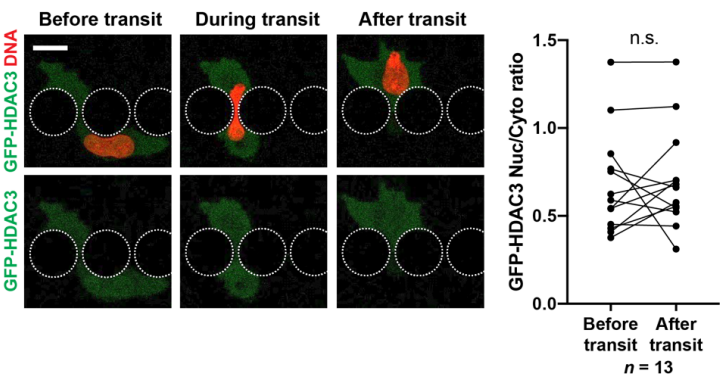

i
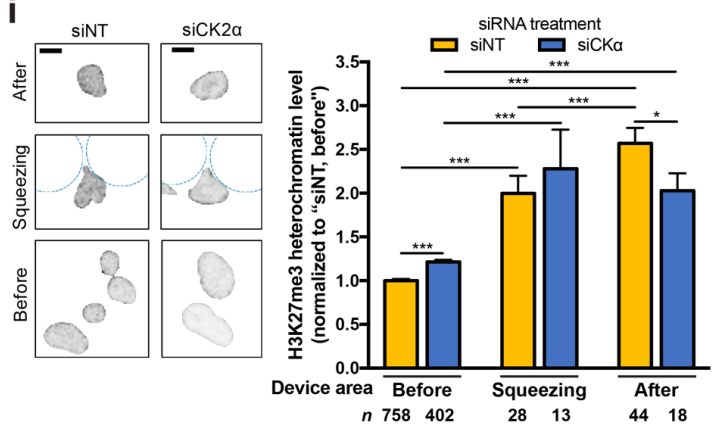

Figure 3: Confined migration-induced heterochromatin formation is dependent on histone modifying enzymes. (a) A simplified schematic of the histone modifying enzymes and the corresponding inhibitors 
involved in the transition between euchromatin state (left, in green) and heterochromatin state (right, in red). (b) Left panel: Representative images of normalized H3K27me3 heterochromatin in HT1080 cells treated with DMSO (vehicle) control or HMTi during confined migration. Scale bars: $10 \mu \mathrm{m}$. Right panel: Quantification of normalized H3K27me3 heterochromatin in cells treated with DMSO (vehicle) control or HMTi during confined migration. All values are normalized to DMSO "before" cells. *** $p<0.001$, twoway ANOVA with Tukey’s multiple comparison test. (c) Left panel: Representative images of normalized H3K27me3 heterochromatin in HT1080 cells treated with DMSO (vehicle) control or pan-HDACi during confined migration. Scale bars: $10 \mu \mathrm{m}$. Right panel: Quantification of normalized H3K27me3 heterochromatin in cells treated with DMSO (vehicle) control or pan-HDACi during confined migration. All values are normalized to DMSO "before" cells. $* * p<0.01$, $* * * p<0.001$, two-way ANOVA with Tukey's multiple comparison test. (d) Left panel: Representative images of normalized H3K27me3 heterochromatin in HT1080 cells treated with DMSO (vehicle) control or HDAC3i during confined migration. Scale bars: $10 \mu \mathrm{m}$. Right panel: Quantification of normalized H3K27me3 heterochromatin in cells treated with DMSO (vehicle) control or HDAC3i during confined migration. All values are normalized to DMSO "before" cells. ${ }^{* *} p<0.01,{ }^{* * *} p<0.001$, two-way ANOVA with Tukey's multiple comparison test. (e) Left panel: Representative images of normalized H3K27me3 heterochromatin in HT1080 cells treated with DMSO (vehicle) control or HMTi + pan-HDACi during confined migration. Scale bars: $10 \mu \mathrm{m}$. Right panel: Quantification of normalized H3K27me3 heterochromatin in cells treated with DMSO (vehicle) control or HMTi + pan-HDACi during confined migration. All values are normalized to DMSO "before" cells. ${ }^{* *} p<0.01, * * * p<0.001$, two-way ANOVA with Tukey's multiple comparison test. (f) Left panel: Representative images of normalized H3K27me3 heterochromatin in HT1080 cells treated with DMSO (vehicle) control or HDMi during confined migration. Scale bars: $10 \mu \mathrm{m}$. Right panel: Quantification of normalized H3K27me3 heterochromatin in cells treated with DMSO (vehicle) control or HDMi during confined migration. All values are normalized to DMSO "before" cells. *** $p<0.001$, two-way ANOVA with Tukey's multiple comparison test. (g) Left panel: Representative image sequences of an HT1080 cell expressing GFP-HDAC3 (green) and stained with SPY555-DNA (red) migrating through a confined constriction. Scale bar: $20 \mu \mathrm{m}$. Right panel: Quantification of GFP-HDAC3 nucleoplasmic-to-cytoplasmic (Nuc/Cyto) ratio changes within an hour of before and after nuclear transit. N.s. not significant based on paired $t$ test $(p=0.531)$. (h) Left panel: Representative images of pSer424-HDAC3 (red) and DAPI (blue) in HT1080 cells during migration through control and confined channels. Scale bars: $10 \mu \mathrm{m}$. Right panel: Quantification of pSer424-HDAC3 intensities during confined migration. All values are normalized to control channels "before" cells. ${ }^{* * *} p<0.001$, two-way ANOVA with Tukey's multiple comparison test. (i) Left panel: Representative images of normalized H3K27me3 heterochromatin in HT1080 cells treated with non-target siRNA (siNT) or CK2 $\alpha$ siRNA $(\operatorname{siCK} 2 \alpha)$ during confined migration. Scale bar: $10 \mu \mathrm{m}$. Right panel: Quantification of normalized H3K27me3 heterochromatin in cells treated with siNT or siCK2 $\alpha$ during confined migration. All values are normalized to siNT "before" cells. * $p<0.05$, *** $p<0.001$, twoway ANOVA with Tukey’s multiple comparison test. Data are presented as mean $\pm \mathrm{SEM}$. 

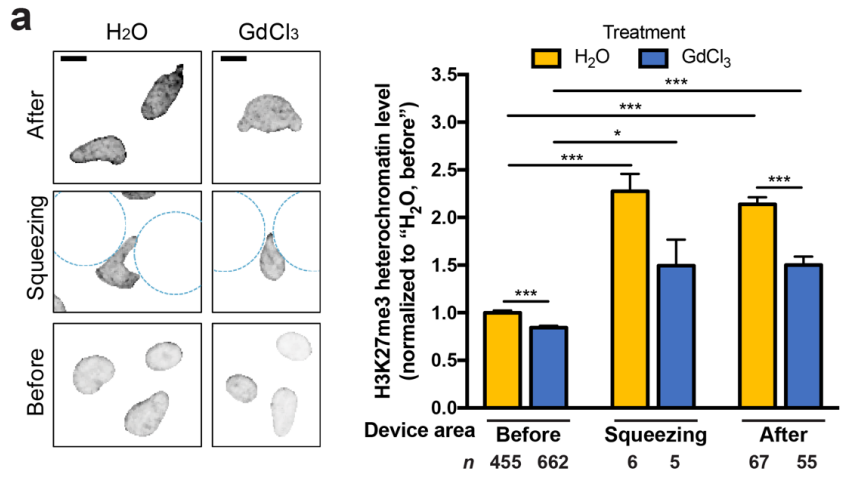

b

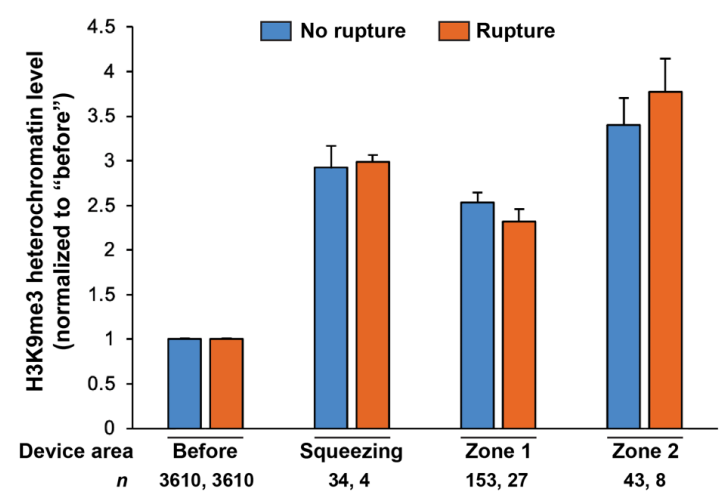

C

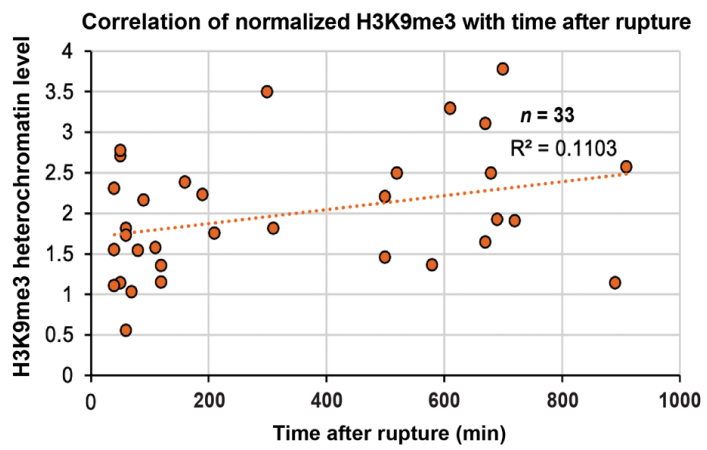

Figure 4: Migration-induced heterochromatin formation is dependent on stretch-sensitive ion channels, but not nuclear envelope rupture. (a) Left panel: Representative images of normalized $\mathrm{H} 3 \mathrm{~K} 27$ me3 heterochromatin in $\mathrm{HT} 1080$ cells treated with $\mathrm{H}_{2} \mathrm{O}$ (vehicle) control or $\mathrm{GdCl}_{3}$ during confined migration. Scale bars: $10 \mu \mathrm{m}$. Right panel: Quantification of normalized H3K27me3 heterochromatin in cells treated with vehicle $\left(\mathrm{H}_{2} \mathrm{O}\right)$ control or $\mathrm{GdCl}_{3}$ during confined migration. All values are normalized to $\mathrm{H}_{2} \mathrm{O}$ "before" cells. ${ }^{*} p<0.05, * * * p<0.001$, two-way ANOVA with Tukey's multiple comparison test. (b) Quantification of normalized H3K9me3 heterochromatin levels in HT1080 cells experienced NE rupture (orange) or not (blue) during confined migration. All "rupture" and "no rupture" heterochromatin levels are normalized the same level in "before" cells. (c) Correlation and linear regression of normalized H3K9me3 heterochromatin levels with time after rupture in HT1080 cells. All values are normalized to "before" cells. Data are presented as mean \pm SEM. 
a
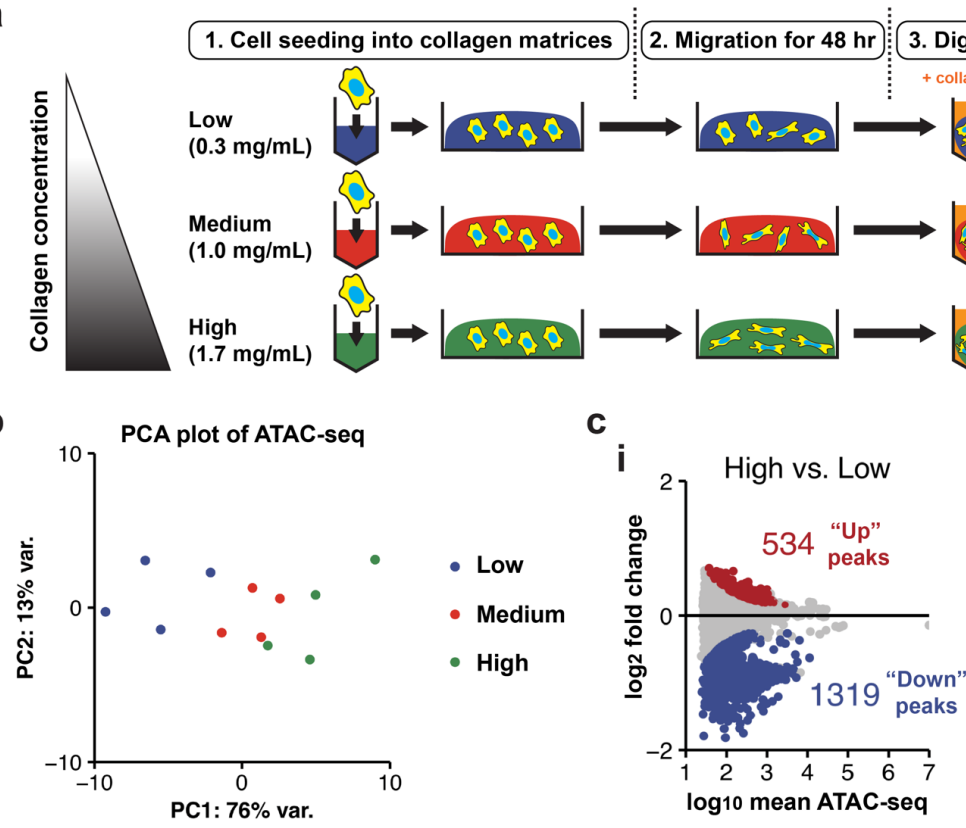

b

d

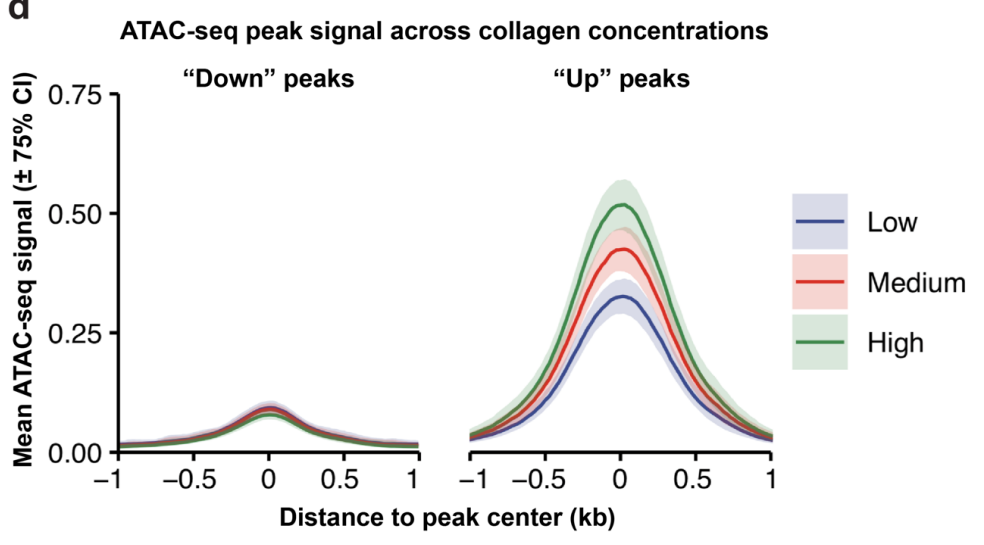

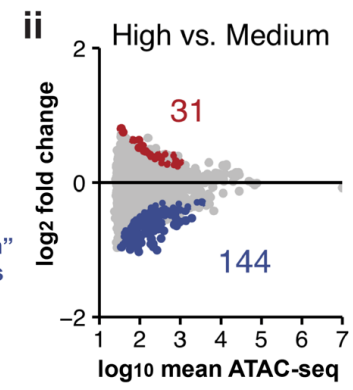

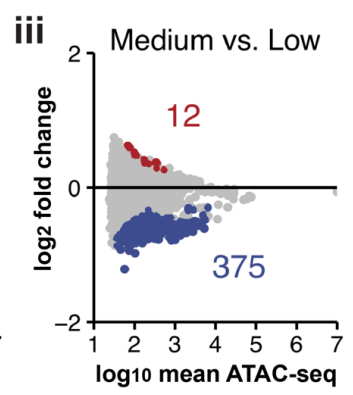

e

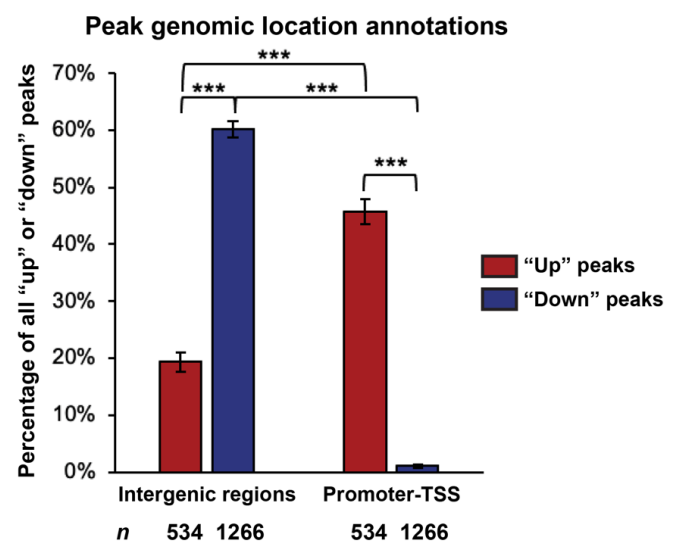

Figure 5: Confined migration alters chromatin accessibility. (a) The workflow of ATAC-seq experiments. HT1080 single cells were seeded into low, medium and high concentration collagen matrices (step 1), and allowed migration for 48 hours (step 2). Collagen matrices were collected, and collagenase was added for digestion and collection of the cells (step 3). ATAC-seq libraries constructed from all samples were sent for sequencing and the results were analyzed (step 4). (b) Principal component analysis (PCA) of all samples. Numbers on PC1 and PC2 axes: percentage of variance (var.) explained by the principal component. (c) MA (logged intensity ratio - mean logged intensities) plots of (i) high versus low, (ii) high versus medium, and (iii) medium versus low concentration collagen samples. Y-axis: $\log _{2}$-fold changes of the peak signal, with the higher concentration collagen samples divided by the lower concentration collagen samples. X-axis: the mean of log ATAC-seq signal across the four biological replicates. Red: up-regulated differentially accessible (DA) peaks ("up" peaks). Purple: down-regulated DA peaks ("down" peaks). Numbers next to the highlighted data points: the numbers of DA peaks. (d) Meta-analysis of ATAC-seq peak signal across collagen concentrations, which compiles the mean of ATAC-seq peak signal in all samples of "down" and "up" peaks (from the high versus low concentration collagen samples). Curves and shaded areas represent mean $\pm 75 \%$ confidence interval (CI). Y-axis: ATAC-seq signal. X-axis: distance to peak center in $\mathrm{kb}$. (e) Annotations of genomic locations that are intergenic or promoter-TSS within "up" and "down" peaks (from the high versus low concentration collagen samples). $* * * p<0.001$, two-way ANOVA with Tukey’s multiple comparison test. Data are presented as mean $\pm \mathrm{SEM}$. 

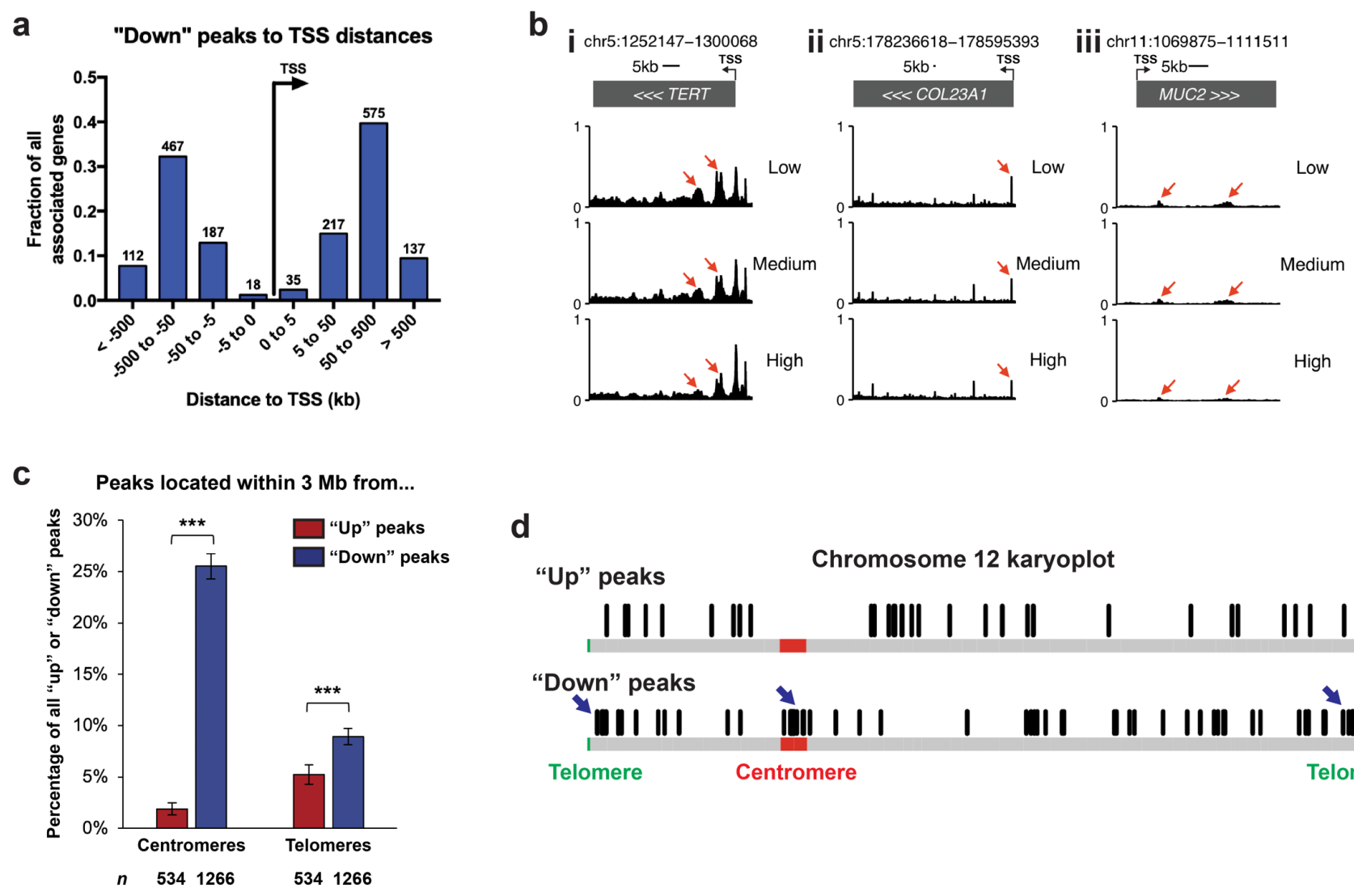

d
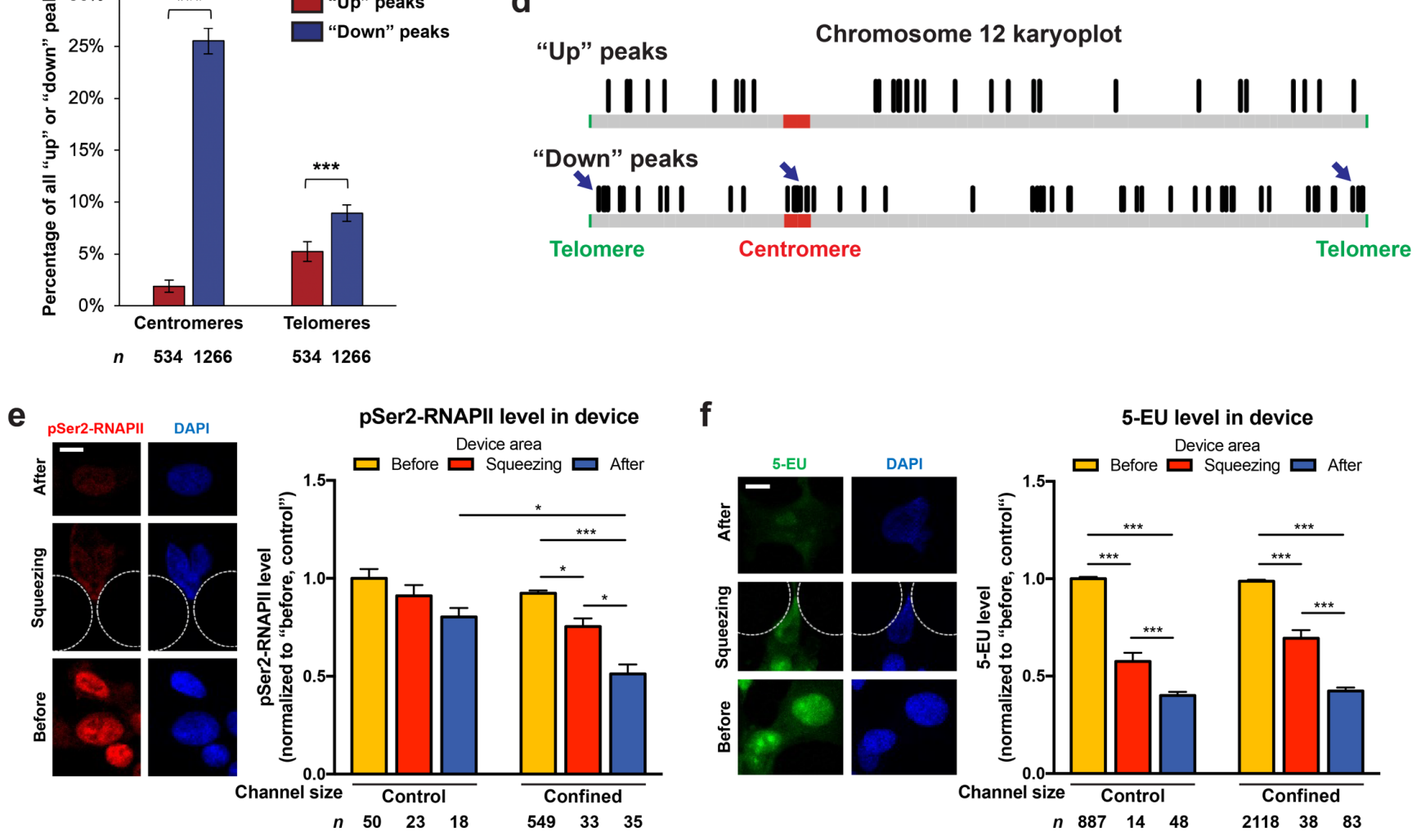

Figure 6: Confined migration decreases intergenic chromatin accessibility near centromeres and telomeres, and global transcription. (a) Distribution of distance between each "down" peak and TSS of its associated genes, calculated by GREAT annotated region-gene associations. Y-axis: the fraction of all associated genes. X-axis: binned distances to TSS in $\mathrm{kb}$. The number on top of each bar: the number of peak-associated genes with distances in that bin. (b) Genome browser shots of three representative genes associated with "down" peaks, (i) TERT, (ii) COL23A1, and (iii) MUC2 ATAC-seq signals in low, medium and high concentration collagen samples. Red arrows: ATAC-seq peaks that are negatively correlated with increasing collagen concentrations. (c) The percentage of "up" and "down" peaks located within $3 \mathrm{Mb}$ from centromeres or telomeres. ${ }^{* * *} p<0.001$, two-way ANOVA with Tukey's multiple comparison test. (d) Representative karyoplot of DA peaks on chromosome 12. Black lines: locations of "up" peaks (top) and "down" peaks (bottom). Red areas: centromeres. Green areas: telomeres. Blue arrows: clustering of "down" peaks near centromeres and telomeres. (e) Left panel: Representative images of pSer2-RNAPII (red) and DAPI (blue) in HT1080 cells during confined migration. Scale bar: $10 \mu \mathrm{m}$. Right panel: Quantification of pSer2-RNAPII intensities in cells migrating through control or confined channels. All values are normalized 
to control channels "before" cells. ${ }^{*} p<0.05, * * * p<0.001$, two-way ANOVA with Tukey's multiple comparison test. (f) Left panel: Representative images of 5-EU (green) and DAPI (blue) in HT1080 cells during confined migration. Scale bar: $10 \mu \mathrm{m}$. Right panel: Quantification of 5-EU intensities in cells migrating through control or confined channels. All values are normalized to control channels "before" cells. $* * * p<0.001$, two-way ANOVA with Tukey's multiple comparison test. Data are presented as mean $\pm \mathrm{SEM}$. 
a

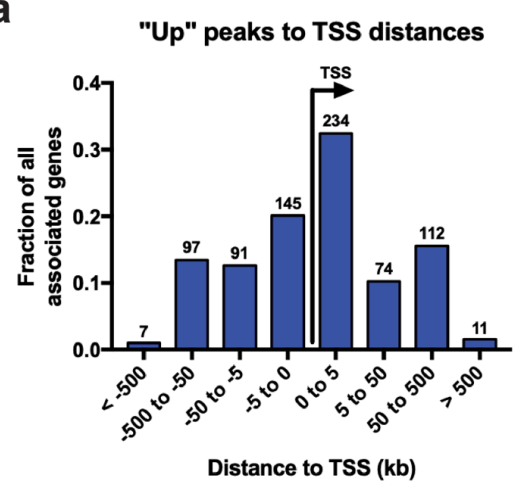

C

Predicted upstream regulators

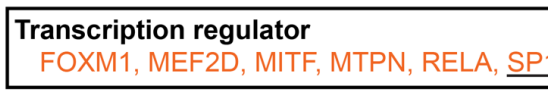

Cytokine
CSF1, CSF2, KITLG, VEGFA

\begin{tabular}{l} 
Kinase \\
INSR, MET, NTRK1, PRKCE \\
\hline
\end{tabular}

Enzyme

APEX1

Other

Jnk, POMC, RETNLB

Transcription regulator

TFAP2A

Transporter

APOE, SFTPA1

microRNA

miR-16-5p (and others with AGCAGCA)

miR-204-5p (and others with UCCCUUU)

d

Nuclear transit time under HMTi

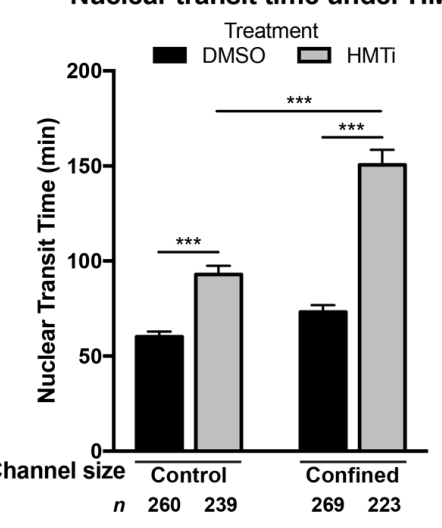

b

i chr11:35657775-35819007 $\quad$ i i chr5:141619876-141641849 $\quad$ ifi chr12:54229942-54285133
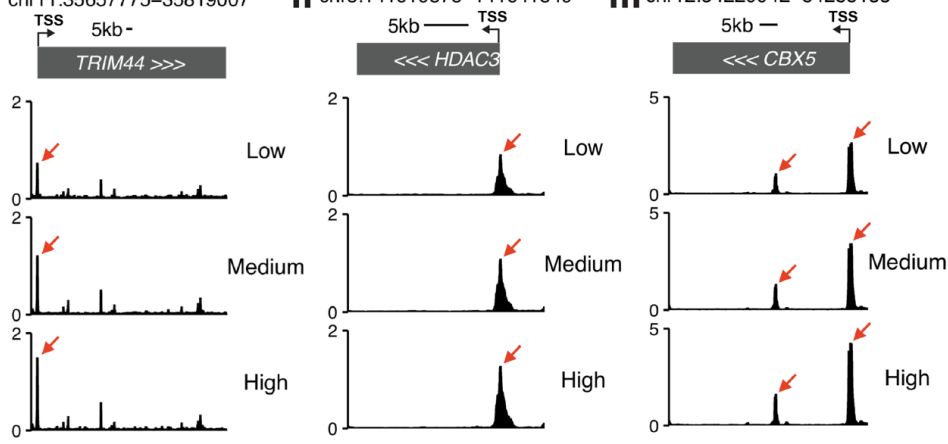

Predicted downstream functions or diseases Migration or tumor invasion-related function Migration/invasion of colorectal cancer cells Cell movement of breast cancer cells Formation of cellular protrusions

Heterochromatin formation-related function

Cell growth or viability-related function Cell proliferation of cervical cancer cells Colony formation of lung cancer cells Cell viability

Synthesis of D-glucose Transactivation

Disease

Development of benign tumor Growth failure or short stature Anemia

Damage or death-related functions DNA damage

Organismal death

e Nuclear transit time under HDMi $\left(37^{\circ} \mathrm{C}\right)$

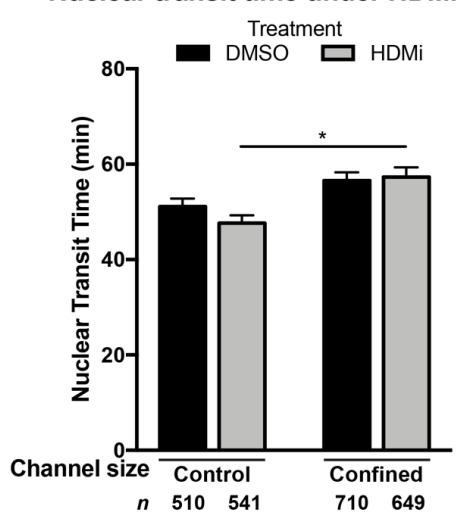

f

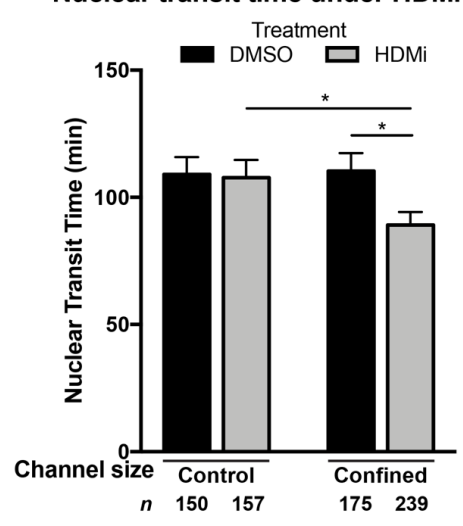

Figure 7: Confined migration increases chromatin accessibility at genes of diverse functions, and confined migration is promoted by heterochromatin formation. (a) Distribution of distance between each "up" peak and TSS of its associated genes, calculated by GREAT annotated region-gene associations. Y-axis: the fraction of all associated genes. X-axis: binned distances to TSS in $\mathrm{kb}$. The number on top of each bar: the number of peak-associated genes with distances in that bin. (b) Genome browser shots of three representative genes associated with "up" peaks, (i) TRIM44, (ii) $H D A C 3$, and (iii) $C B X 5$ (which encodes HP1 $\alpha$ ) ATAC-seq signals in low, medium and high concentration collagen samples. Red arrows: ATACseq peaks that are positively correlated with increasing collagen concentrations. (c) Predicted network of 
upstream regulators (left) and downstream functions (right) of genes associated with "up" peaks, as calculated by IPA (Ingenuity Pathway Analysis). Orange indicates activation, whereas blue indicates inhibition. Underlined: SP1. Red box: activation of cell migration or tumor invasion-related functions. Green box: activation of heterochromatin formation-related function. For the full network, please refer to Suppl. Figure 9. (d) Quantification of HT1080 cells nuclear transit time through control or confined channels, under DMSO (vehicle) or HMTi treatment. ${ }^{* * *} p<0.001$, Kruskal-Wallis H test with Dunn's multiple comparison test. (e) Quantification of HT1080 cells nuclear transit time through control or confined channels, under DMSO (vehicle) or HDMi treatment. * $p<0.05$, Kruskal-Wallis H test with Dunn's multiple comparison test. (f) Quantification of HT1080 cells nuclear transit time through control or confined channels, under DMSO (vehicle) or HDMi treatment under mild hypothermia stress (at $33^{\circ} \mathrm{C}$ ). ${ }^{*} p<0.05$, KruskalWallis H test with Dunn's multiple comparison test. Data are presented as mean $\pm \mathrm{SEM}$. 
Table 1: Gene Ontology (GO) Biological Process terms enrichment of genes associated with upregulated DA peaks in high concentration collagen samples. ${ }^{*}$ Chromatin or gene silencing-related GO terms.

\begin{tabular}{|l|l|l|}
\hline GO term & Binominal rank & Binominal $\boldsymbol{p}$-value \\
\hline Chromatin silencing* & 1 & $2.2103 \mathrm{e}-11$ \\
\hline Nucleosome assembly* & 2 & $4.9680 \mathrm{e}-9$ \\
\hline Negative regulation of gene expression, epigenetic* & 3 & $1.0730 \mathrm{e}-8$ \\
\hline Chromatin assembly* & 5 & $3.5672 \mathrm{e}-8$ \\
\hline DNA packaging* & 7 & $4.0250 \mathrm{e}-8$ \\
\hline Gene silencing* & 8 & $4.2400 \mathrm{e}-8$ \\
\hline Nucleosome organization* & 9 & $1.0150 \mathrm{e}-7$ \\
\hline Chromosome organization* & 10 & $1.4824 \mathrm{e}-7$ \\
\hline Chromatin assembly or disassembly* & 12 & $2.8667 \mathrm{e}-7$ \\
\hline Regulation of gene expression, epigenetic* & 14 & $3.9674 \mathrm{e}-7$ \\
\hline DNA conformation change & 15 & $2.3367 \mathrm{e}-6$ \\
\hline DNA integrity checkpoint & 17 & $4.9484 \mathrm{e}-6$ \\
\hline Cell cycle checkpoint & 18 & $5.6378 \mathrm{e}-6$ \\
\hline Protein-DNA complex assembly* & 19 & $5.7591 \mathrm{e}-6$ \\
\hline DNA damage checkpoint & 20 & $8.9630 \mathrm{e}-6$ \\
\hline Protien-DNA complex subunit organization* & 24 & $1.6041 \mathrm{e}-5$ \\
\hline Protein heterotetramerization* & 26 & $2.6928 \mathrm{e}-5$ \\
\hline Regulation of megakaryocyte differentiation & 27 & $3.4551 \mathrm{e}-5$ \\
\hline Positive regulation of cell cycle & 28 & $3.6975 \mathrm{e}-5$ \\
\hline Positive regulation of gene expression, epigenetic* & 33 & $4.4949 \mathrm{e}-5$ \\
\hline Positive regulation of cell cycle process & 36 & $5.0241 \mathrm{e}-5$ \\
\hline
\end{tabular}

Table 2: Gene Ontology (GO) Cellular Component terms enrichment of genes associated with upregulated DA peaks in high concentration collagen samples. *Chromatin or gene silencing-related GO terms.

\begin{tabular}{|l|l|l|}
\hline GO term & Binominal rank & Binominal $\boldsymbol{p}$-value \\
\hline DNA packaging complex* & 1 & $6.5383 \mathrm{e}-14$ \\
\hline Nucleosome* & 2 & $1.5848 \mathrm{e}-13$ \\
\hline Protein-DNA complex* & 8 & $8.2620 \mathrm{e}-8$ \\
\hline Nuclear nucleosome* & 11 & $1.7191 \mathrm{e}-5$ \\
\hline Chromosome, telomeric region* & 13 & $5.8580 \mathrm{e}-5$ \\
\hline Nuclear chromosome, telomeric region* & 14 & $6.0764 \mathrm{e}-5$ \\
\hline Chromosomal region* & 15 & $8.0535 \mathrm{e}-5$ \\
\hline
\end{tabular}


Table 3: Canonical pathways of genes associated with up-regulated DA peaks in high concentration collagen samples. Only pathways with $-\log (p$-value $)>1.30(p$-value $<0.05)$ and $\mid Z$-score $\mid($ if known $) \geq 1$ are shown. NA, not available. $\uparrow$ Cell migration or tumor invasion-related pathways. *Histone methylation or heterochromatin formation-related pathways.

\begin{tabular}{|c|c|c|c|}
\hline Ingenuity Canonical Pathways & Rank & -log(p-value $)$ & Z-score \\
\hline NRF2-mediated Oxidative Stress Response $\dagger$ & 1 & 3.94 & 2.333 \\
\hline Antioxidant Action of Vitamin C & 2 & 3.22 & -2.449 \\
\hline Spliceosomal Cycle & 3 & 2.86 & 2.236 \\
\hline Kinetochore Metaphase Signaling Pathway & 4 & 2.74 & 1.134 \\
\hline Protein Ubiquitination Pathway & 5 & 2.54 & NA \\
\hline Superpathway of Methionine Degradation* & 6 & 2.44 & 2 \\
\hline mTOR Signaling $\dagger$ & 7 & 2.44 & 1.633 \\
\hline p38 MAPK Signaling $\dagger$ & 8 & 2.36 & 1.342 \\
\hline Estrogen Receptor Signaling $\dagger$ & 9 & 2.32 & 2.887 \\
\hline Phospholipases & 10 & 2.28 & 2.236 \\
\hline Thioredoxin Pathway & 11 & 2.23 & NA \\
\hline Hypoxia Signaling in the Cardiovascular System & 12 & 2.05 & NA \\
\hline Cell Cycle: G2/M DNA Damage Checkpoint Regulation & 13 & 2 & -2 \\
\hline Toll-like Receptor Signaling & 14 & 2 & NA \\
\hline CD27 Signaling in Lymphocytes & 15 & 1.88 & 2 \\
\hline Endothelin-1 Signaling $\dagger$ & 16 & 1.78 & 2.828 \\
\hline Role of PKR in Interferon Induction and Antiviral Response & 17 & 1.78 & 1.342 \\
\hline Glycogen Degradation II & 18 & 1.76 & NA \\
\hline IL-1 Signaling $\dagger$ & 19 & 1.67 & 1.342 \\
\hline DNA Methylation and Transcriptional Repression Signaling* & 20 & 1.66 & NA \\
\hline Glycogen Degradation III & 21 & 1.63 & NA \\
\hline TGF- $\beta$ Signaling $\dagger$ & 22 & 1.6 & 1.342 \\
\hline Insulin Secretion Signaling Pathway & 23 & 1.58 & 3 \\
\hline Choline Biosynthesis III & 24 & 1.57 & NA \\
\hline Mitotic Roles of Polo-Like Kinase & 25 & 1.57 & NA \\
\hline Pyridoxal 5'-phosphate Salvage Pathway $\dagger$ & 26 & 1.57 & NA \\
\hline UVA-Induced MAPK Signaling & 27 & 1.56 & NA \\
\hline Remodeling of Epithelial Adherens Junctions $\dagger$ & 28 & 1.53 & NA \\
\hline GNRH Signaling & 29 & 1.51 & 2.646 \\
\hline April Mediated Signaling & 30 & 1.5 & NA \\
\hline IL-10 Signaling $\dagger$ & 31 & 1.49 & NA \\
\hline NER Pathway & 32 & 1.48 & -1.342 \\
\hline B Cell Activating Factor Signaling & 33 & 1.48 & NA \\
\hline IGF-1 Signaling $\dagger$ & 34 & 1.47 & NA \\
\hline ERK5 Signaling $\dagger$ & 35 & 1.45 & 2 \\
\hline Endocannabinoid Cancer Inhibition Pathway & 36 & 1.42 & 1.633 \\
\hline Pancreatic Adenocarcinoma Signaling $\dagger$ & 37 & 1.39 & NA \\
\hline iNOS Signaling $\dagger$ & 38 & 1.37 & NA \\
\hline PFKFB4 Signaling Pathway $\dagger$ & 39 & 1.35 & NA \\
\hline Endocannabinoid Developing Neuron Pathway & 40 & 1.31 & 1 \\
\hline Renal Cell Carcinoma Signaling $\dagger$ & 41 & 1.31 & NA \\
\hline Chemokine Signaling $\dagger$ & 42 & 1.31 & 2 \\
\hline
\end{tabular}




\section{References}

1. Scarpa, E. \& Mayor, R. Collective cell migration in development. J Cell Biol 212, 143-155 (2016).

2. Luster, A. D., Alon, R. \& von Andrian, U. H. Immune cell migration in inflammation: present and future therapeutic targets. Nature Immunology 6, 1182-1190 (2005).

3. Chaffer, C. L. \& Weinberg, R. A. A perspective on cancer cell metastasis. Science 331, 1559-1564 (2011).

4. Lambert, A. W., Pattabiraman, D. R. \& Weinberg, R. A. Emerging Biological Principles of Metastasis. Cell 168, 670-691 (2017).

5. Kameritsch, P. \& Renkawitz, J. Principles of Leukocyte Migration Strategies. Trends Cell Biol 30, 818-832 (2020).

6. Weigelin, B., Bakker, G.-J. \& Friedl, P. Intravital third harmonic generation microscopy of collective melanoma cell invasion: Principles of interface guidance and microvesicle dynamics. Intravital 1, 3243 (2012).

7. Paul, C. D., Mistriotis, P. \& Konstantopoulos, K. Cancer cell motility: lessons from migration in confined spaces. Nature Reviews Cancer 17, 131-140 (2017).

8. Lammerding, J. Mechanics of the Nucleus. Compr Physiol 1, 783-807 (2011).

9. Swift, J. et al. Nuclear Lamin-A Scales with Tissue Stiffness and Enhances Matrix-Directed Differentiation. Science 341, (2013).

10. Denais, C. M. et al. Nuclear envelope rupture and repair during cancer cell migration. Science $\mathbf{3 5 2}$, 353-358 (2016).

11. Irianto, J. et al. Nuclear constriction segregates mobile nuclear proteins away from chromatin. $M o l$ Biol Cell 27, 4011-4020 (2016).

12. Raab, M. et al. ESCRT III repairs nuclear envelope ruptures during cell migration to limit DNA damage and cell death. Science 352, 359-362 (2016).

13. Shah, P. et al. Nuclear Deformation Causes DNA Damage by Increasing Replication Stress. Current Biology (2020) doi:10.1016/j.cub.2020.11.037.

14. Jacobson, E. C. et al. Migration through a small pore disrupts inactive chromatin organization in neutrophil-like cells. BMC Biol 16, 142 (2018).

15. Golloshi, R. et al. Constricted migration is associated with stable 3D genome structure differences in melanoma cells. bioRxiv 856583 (2020) doi:10.1101/856583.

16. Janssen, A., Colmenares, S. U. \& Karpen, G. H. Heterochromatin: Guardian of the Genome. Annu Rev Cell Dev Biol 34, 265-288 (2018).

17. Strålfors, A. \& Ekwall, K. Heterochromatin and Euchromatin-Organization, Boundaries, and Gene Regulation. in Reviews in Cell Biology and Molecular Medicine (American Cancer Society, 2011). doi:10.1002/3527600906.mcb.200400018.pub2.

18. Bannister, A. J. \& Kouzarides, T. Regulation of chromatin by histone modifications. Cell Research 21, 381-395 (2011).

19. Rose, N. R. \& Klose, R. J. Understanding the relationship between DNA methylation and histone lysine methylation. Biochimica et Biophysica Acta (BBA) - Gene Regulatory Mechanisms 1839, 13621372 (2014).

20. Lennartsson, A. \& Ekwall, K. Histone modification patterns and epigenetic codes. Biochimica et Biophysica Acta (BBA) - General Subjects 1790, 863-868 (2009).

21. Gerlitz, G. The Emerging Roles of Heterochromatin in Cell Migration. Front. Cell Dev. Biol. 8, (2020).

22. Gerlitz, G. \& Bustin, M. Efficient cell migration requires global chromatin condensation. $J$ Cell Sci 123, 2207-2217 (2010).

23. Liu, L. et al. Chromatin organization regulated by EZH2-mediated H3K27me3 is required for OPNinduced migration of bone marrow-derived mesenchymal stem cells. The International Journal of Biochemistry \& Cell Biology 96, 29-39 (2018). 
24. Zhang, B. et al. Increased nuclear stiffness via FAK-ERK1/2 signaling is necessary for synthetic mechano-growth factor E peptide-induced tenocyte migration. Sci Rep 6, (2016).

25. Zhang, X. et al. Integrin $\alpha 4 \beta 1$ controls G9a activity that regulates epigenetic changes and nuclear properties required for lymphocyte migration. Nucleic Acids Res 44, 3031-3044 (2016).

26. Segal, T., Salmon-Divon, M. \& Gerlitz, G. The Heterochromatin Landscape in Migrating Cells and the Importance of H3K27me3 for Associated Transcriptome Alterations. Cells 7, (2018).

27. Wang, P. et al. WDR5 modulates cell motility and morphology and controls nuclear changes induced by a $3 \mathrm{D}$ environment. PNAS 115, 8581-8586 (2018).

28. Fischer, T., Hayn, A. \& Mierke, C. T. Effect of Nuclear Stiffness on Cell Mechanics and Migration of Human Breast Cancer Cells. Front Cell Dev Biol 8, 393 (2020).

29. Stephens, A. D. et al. Chromatin histone modifications and rigidity affect nuclear morphology independent of lamins. Mol Biol Cell 29, 220-233 (2018).

30. Damodaran, K. et al. Compressive force induces reversible chromatin condensation and cell geometry-dependent transcriptional response. $M B o C$ 29, 3039-3051 (2018).

31. Nava, M. M. et al. Heterochromatin-Driven Nuclear Softening Protects the Genome against Mechanical Stress-Induced Damage. Cell 181, 800-817.e22 (2020).

32. Wolf, K. et al. Physical limits of cell migration: control by ECM space and nuclear deformation and tuning by proteolysis and traction force. J Cell Biol 201, 1069-1084 (2013).

33. Karoutas, A. et al. The NSL complex maintains nuclear architecture stability via lamin A/C acetylation. Nature Cell Biology 21, 1248-1260 (2019).

34. Davidson, P. M., Sliz, J., Isermann, P., Denais, C. \& Lammerding, J. Design of a microfluidic device to quantify dynamic intra-nuclear deformation during cell migration through confining environments. Integrative Biology 7, 1534-1546 (2015).

35. Lomakin, A. J. et al. The nucleus acts as a ruler tailoring cell responses to spatial constraints. Science 370, (2020).

36. Venturini, V. et al. The nucleus measures shape changes for cellular proprioception to control dynamic cell behavior. Science 370, (2020).

37. Boros, J., Arnoult, N., Stroobant, V., Collet, J.-F. \& Decottignies, A. Polycomb Repressive Complex 2 and H3K27me3 Cooperate with H3K9 Methylation To Maintain Heterochromatin Protein $1 \alpha$ at Chromatin. Mol Cell Biol 34, 3662-3674 (2014).

38. Cheutin, T. et al. Maintenance of stable heterochromatin domains by dynamic HP1 binding. Science 299, 721-725 (2003).

39. Schmiedeberg, L., Weisshart, K., Diekmann, S., Meyer zu Hoerste, G. \& Hemmerich, P. High- and Low-mobility Populations of HP1 in Heterochromatin of Mammalian Cells. Mol Biol Cell 15, 28192833 (2004).

40. Miranda, T. B. et al. DZNep Is a Global Histone Methylation Inhibitor that Reactivates Developmental Genes Not Silenced by DNA Methylation. Mol Cancer Ther 8, 1579-1588 (2009).

41. Vigushin, D. M. et al. Trichostatin A is a histone deacetylase inhibitor with potent antitumor activity against breast cancer in vivo. Clin Cancer Res 7, 971-976 (2001).

42. Alisafaei, F., Jokhun, D. S., Shivashankar, G. V. \& Shenoy, V. B. Regulation of nuclear architecture, mechanics, and nucleocytoplasmic shuttling of epigenetic factors by cell geometric constraints. Proc Natl Acad Sci U S A 116, 13200-13209 (2019).

43. Malvaez, M. et al. HDAC3-selective inhibitor enhances extinction of cocaine-seeking behavior in a persistent manner. Proc Natl Acad Sci US A 110, 2647-2652 (2013).

44. Parrish, J. K. et al. The Jumonji-domain histone demethylase inhibitor JIB-04 deregulates oncogenic programs and increases DNA damage in Ewing Sarcoma, resulting in impaired cell proliferation and survival, and reduced tumor growth. Oncotarget 9, 33110-33123 (2018).

45. Zhang, X. et al. Histone deacetylase 3 (HDAC3) activity is regulated by interaction with protein serine/threonine phosphatase 4. Genes Dev 19, 827-839 (2005).

46. Litchfield, D. W. Protein kinase CK2: structure, regulation and role in cellular decisions of life and death. Biochem J 369, 1-15 (2003). 
47. Poleshko, A. et al. Genome-nuclear lamina interactions regulate cardiac stem cell lineage restriction. Cell 171, 573-587.e14 (2017).

48. Demmerle, J., Koch, A. J. \& Holaska, J. M. The Nuclear Envelope Protein Emerin Binds Directly to Histone Deacetylase 3 (HDAC3) and Activates HDAC3 Activity. J Biol Chem 287, 22080-22088 (2012).

49. van Steensel, B. \& Belmont, A. S. Lamina-associated domains: links with chromosome architecture, heterochromatin and gene repression. Cell 169, 780-791 (2017).

50. Ranade, D., Pradhan, R., Jayakrishnan, M., Hegde, S. \& Sengupta, K. Lamin A/C and Emerin depletion impacts chromatin organization and dynamics in the interphase nucleus. BMC Molecular and Cell Biology 20, 11 (2019).

51. Dechat, T., Adam, S. A. \& Goldman, R. D. Nuclear Lamins and Chromatin: When Structure Meets Function. Adv Enzyme Regul 49, 157-166 (2009).

52. Bronshtein, I. et al. Loss of lamin A function increases chromatin dynamics in the nuclear interior. Nat Commun 6, 8044 (2015).

53. Caldwell, R. A., Clemo, H. F. \& Baumgarten, C. M. Using gadolinium to identify stretch-activated channels: technical considerations. American Journal of Physiology-Cell Physiology 275, C619-C621 (1998).

54. Buenrostro, J., Wu, B., Chang, H. \& Greenleaf, W. ATAC-seq: A Method for Assaying Chromatin Accessibility Genome-Wide. Curr Protoc Mol Biol 109, 21.29.1-21.29.9 (2015).

55. Tsompana, M. \& Buck, M. J. Chromatin accessibility: a window into the genome. Epigenetics \& Chromatin 7, 33 (2014).

56. Corces, M. R. et al. An improved ATAC-seq protocol reduces background and enables interrogation of frozen tissues. Nature Methods 14, 959-962 (2017).

57. McLean, C. Y. et al. GREAT improves functional interpretation of cis-regulatory regions. Nat Biotechnol 28, 495-501 (2010).

58. Allshire, R. C. \& Madhani, H. D. Ten principles of heterochromatin formation and function. Nat Rev Mol Cell Biol 19, 229-244 (2018).

59. Wang, J., Lawry, S. T., Cohen, A. L. \& Jia, S. Chromosome boundary elements and regulation of heterochromatin spreading. Cell. Mol. Life Sci. 71, 4841-4852 (2014).

60. Levy-Sakin, M. et al. Genome maps across 26 human populations reveal population-specific patterns of structural variation. Nature Communications 10, 1025 (2019).

61. Slotkin, R. K. \& Martienssen, R. Transposable elements and the epigenetic regulation of the genome. Nature Reviews Genetics 8, 272-285 (2007).

62. Kapusta, A. et al. Transposable elements are major contributors to the origin, diversification, and regulation of vertebrate long noncoding RNAs. PLoS Genet 9, e1003470 (2013).

63. Lynch, V. J. et al. Ancient transposable elements transformed the uterine regulatory landscape and transcriptome during the evolution of mammalian pregnancy. Cell Rep 10, 551-561 (2015).

64. Bersani, F. et al. Pericentromeric satellite repeat expansions through RNA-derived DNA intermediates in cancer. Proc Natl Acad Sci U S A 112, 15148-15153 (2015).

65. Bennett, E. A. et al. Active Alu retrotransposons in the human genome. Genome Res 18, 1875-1883 (2008).

66. Bartkowiak, B. \& Greenleaf, A. L. Phosphorylation of RNAPII. Transcription 2, 115-119 (2011).

67. Jao, C. Y. \& Salic, A. Exploring RNA transcription and turnover in vivo by using click chemistry. PNAS 105, 15779-15784 (2008).

68. Krämer, A., Green, J., Pollard, J., Jr \& Tugendreich, S. Causal analysis approaches in Ingenuity Pathway Analysis. Bioinformatics 30, 523-530 (2014).

69. Irianto, J. et al. DNA damage follows repair factor depletion and portends genome variation in cancer cells after pore migration. Curr Biol 27, 210-223 (2017).

70. Moriarty, R. A. \& Stroka, K. M. Physical confinement alters sarcoma cell cycle progression and division. Cell Cycle 17, 2360-2373 (2018). 
71. Xia, Y. et al. Rescue of DNA damage after constricted migration reveals a mechano-regulated threshold for cell cycle. Journal of Cell Biology 218, 2545-2563 (2019).

72. Jungert, K. et al. Smad-Sp1 complexes mediate TGFbeta-induced early transcription of oncogenic Smad7 in pancreatic cancer cells. Carcinogenesis 27, 2392-2401 (2006).

73. Lang, C. et al. SMAD3/SP1 complex-mediated constitutive active loop between lncRNA PCAT7 and TGF- $\beta$ signaling promotes prostate cancer bone metastasis. Mol Oncol 14, 808-828 (2020).

74. Klemm, S. L., Shipony, Z. \& Greenleaf, W. J. Chromatin accessibility and the regulatory epigenome. Nature Reviews Genetics 20, 207-220 (2019).

75. Pliner, H. A. et al. Cicero Predicts cis-Regulatory DNA Interactions from Single-Cell Chromatin Accessibility Data. Mol Cell 71, 858-871.e8 (2018).

76. Rosenbloom, K. R. et al. ENCODE data in the UCSC Genome Browser: year 5 update. Nucleic Acids Res 41, D56-63 (2013).

77. Schoenfelder, S. \& Fraser, P. Long-range enhancer-promoter contacts in gene expression control. Nat Rev Genet 20, 437-455 (2019).

78. van Arensbergen, J., van Steensel, B. \& Bussemaker, H. J. In search of the determinants of enhancerpromoter interaction specificity. Trends Cell Biol 24, 695-702 (2014).

79. Fu, Y., Chin, L. K., Bourouina, T., Liu, A. Q. \& VanDongen, A. M. J. Nuclear deformation during breast cancer cell transmigration. Lab Chip 12, 3774-3778 (2012).

80. Krause, M. et al. Cell migration through three-dimensional confining pores: speed accelerations by deformation and recoil of the nucleus. Philosophical Transactions of the Royal Society B: Biological Sciences 374, 20180225 (2019).

81. Davidson, P. M., Denais, C., Bakshi, M. C. \& Lammerding, J. Nuclear deformability constitutes a rate-limiting step during cell migration in 3-D environments. Cell Mol Bioeng 7, 293-306 (2014).

82. Lautscham, L. A. et al. Migration in Confined 3D Environments Is Determined by a Combination of Adhesiveness, Nuclear Volume, Contractility, and Cell Stiffness. Biophys J 109, 900-913 (2015).

83. Fulbert, C., Gaude, C., Sulpice, E., Chabardès, S. \& Ratel, D. Moderate hypothermia inhibits both proliferation and migration of human glioblastoma cells. J Neurooncol 144, 489-499 (2019).

84. Kalamida, D. et al. Fever-Range Hyperthermia vs. Hypothermia Effect on Cancer Cell Viability, Proliferation and HSP90 Expression. PLoS One 10, (2015).

85. Jiang, Y. et al. Common fragile sites are characterized by histone hypoacetylation. Hum Mol Genet 18, 4501-4512 (2009).

86. Kurashima, K. et al. SMARCA4 deficiency-associated heterochromatin induces intrinsic DNA replication stress and susceptibility to ATR inhibition in lung adenocarcinoma. NAR Cancer 2, (2020).

87. Lambert, S. \& Carr, A. M. Impediments to replication fork movement: stabilisation, reactivation and genome instability. Chromosoma 122, 33-45 (2013).

88. Nikolov, I. \& Taddei, A. Linking replication stress with heterochromatin formation. Chromosoma 125, 523-533 (2016).

89. Chang, W., Worman, H. J. \& Gundersen, G. G. Accessorizing and anchoring the LINC complex for multifunctionality. Journal of Cell Biology 208, 11-22 (2015).

90. Crisp, M. et al. Coupling of the nucleus and cytoplasm: role of the LINC complex. J Cell Biol 172, 41-53 (2006).

91. Peng, Q. et al. Coordinated histone modifications and chromatin reorganization in a single cell revealed by FRET biosensors. PNAS 115, E11681-E11690 (2018).

92. Sato, Y. et al. Genetically encoded system to track histone modification in vivo. Scientific Reports $\mathbf{3}$, $2436(2013)$.

93. Elacqua, J. J., McGregor, A. L. \& Lammerding, J. Automated analysis of cell migration and nuclear envelope rupture in confined environments. PLOS ONE 13, e0195664 (2018).

94. Cross, V. L. et al. Dense type I collagen matrices that support cellular remodeling and microfabrication for studies of tumor angiogenesis and vasculogenesis in vitro. Biomaterials $\mathbf{3 1}$, 8596-8607 (2010). 